\title{
Unipotent vector bundles and higher-order non-holomorphic Eisenstein series
}

\author{
Jay Jorgenson, Cormac O’Sullivan ${ }^{\dagger}$
}

7 October 2006

\begin{abstract}
Higher-order non-holomorphic Eisenstein series associated to a Fuchsian group $\Gamma$ are defined by twisting the series expansion for classical non-holomorphic Eisenstein series by powers of modular symbols. Their functional identities include multiplicative and additive factors, making them distinct from classical Eisenstein series. In this article we prove the meromorphic continuation of these series and establish their functional equations which relate values at $s$ and $1-s$. In addition, we construct high rank vector bundles $\mathcal{V}$ from certain unipotent representations $\pi$ of $\Gamma$ and show that higher-order non-holomorphic Eisenstein series can be viewed as components of certain eigensections, $\mathbb{E}$, of $\mathcal{V}$. With this viewpoint the functional identities of these higher-order series are formally identical to the classical case. Going further, we prove bounds for the Fourier coefficients of the higher-order non-holomorphic Eisenstein series.
\end{abstract}

\section{Introduction}

Let $\Gamma$ be any Fuchsian group of the first kind which acts on the hyperbolic upper half-space $\mathbf{H}$ such that the quotient $\Gamma \backslash \mathbf{H}$ has finite volume yet is non-compact. Following the notation from [11], let us fix representatives for the finite number of $\Gamma$-inequivalent cusps, label them $\mathfrak{a}, \mathfrak{b}, \ldots$, and use the scaling matrices $\sigma_{\mathfrak{a}}, \sigma_{\mathfrak{b}}, \ldots$ to give local coordinates near these cusps. Define the subgroup $\Gamma_{\mathfrak{a}}$ to be the elements of $\Gamma$ which fix the cusps equivalent to a. Let $S_{2}(\Gamma)$ be the space of holomorphic cusp forms of weight 2 with respect to $\Gamma$. For $f, g \in S_{2}(\Gamma)$ and $m, n \geqslant 0$, we define, following [7], [8], and [17], the higher-order non-holomorphic Eisenstein series associated to the forms $f$ and $g$, character $\chi$ and cusp $\mathfrak{a}$ by the series

$$
E_{\mathfrak{a}}^{m, n}(z, s ; f, g, \chi)=\sum_{\gamma \in \Gamma_{\mathfrak{a}} \backslash \Gamma} \chi(\gamma)\langle\gamma, f\rangle^{m} \overline{\langle\gamma, g\rangle}^{n} \operatorname{Im}\left({\sigma_{\mathfrak{a}}}^{-1} \gamma z\right)^{s}
$$

where the modular symbols are defined by

$$
\langle\gamma, f\rangle=\int_{z_{0}}^{\gamma z_{0}} f(w) d w,\langle\gamma, g\rangle=\int_{z_{0}}^{\gamma z_{0}} g(w) d w ;
$$

observe that the modular symbols are independent of $z_{0} \in \mathbf{H}$ by Cauchy's theorem since $f$ and $g$ are holomorphic and weight 2 . We will discuss the exact meaning of order in section 3.1. Throughout this article, we will consider the forms $f, g$ and character $\chi$ to be fixed, hence we will abbreviate the notation and just write $E_{\mathfrak{a}}^{m, n}(z, s)$.

As will be shown, the series (1.1) converges absolutely for $\operatorname{Re}(s)>1$ and $z \in \mathbf{H}$. One of the main results of this article is to prove the meromorphic continuation of $E_{\mathfrak{a}}^{m, n}(z, s)$ to all $s$ in $\mathbf{C}$. The continuation of (1.1) has already been addressed in [18] and [19] using perturbation theory. Our new proof continues the extension of Selberg's method [21] to higher-order forms that appears in [17], [12]. It has the advantage of naturally giving strong bounds on both the Fourier coefficients of $E_{\mathfrak{a}}^{m, n}(z, s)$ and its growth in $z$. We require these bounds for our demonstration of the functional equation, relating values of higher-order Eisenstein series in the left and right $s$-planes; the determination of the functional equation of (1.1) is one of the main

\footnotetext{
*The first author was partially supported by PSC-CUNY Research Award No. 67280-00-36 and NSF Grant 05-03669.

$\dagger$ The second author was partially supported by PSC-CUNY Research Award No. 67478-00 36 and PSC-CUNY Collaborative Award No. 80209-04 11.
} 
new features of the present article. Finally, we formulate our results using the language and notation of vector bundles, which shows that the Eisenstein series (1.1) naturally can be viewed as components of eigensections of certain vector bundles.

The properties of $E_{\mathfrak{a}}^{m, n}$ are developed here with two new applications in mind. First, it is well known that the Kronecker limit formula for the classical Eisenstein series $E_{\mathfrak{a}}^{0,0}$ (usually written as just $E_{\mathfrak{a}}$ ) produces Dedekind sums. In our forthcoming work [13], we find that $E_{\mathfrak{a}}^{1,1}$ yields new types of Dedekind sums with interesting arithmetic. No such sums were found for $E_{\mathfrak{a}}^{1,0}$ though the explicit Kronecker limit formula for that case was revealed in [12] which included new families of $L$-functions associated to the Fourier coefficients of modular forms and classical Kronecker limit functions. Second, in current work of the second author and Imamoḡlu, a natural inner product for the space of higher-order weight $k$ forms is found. The usual Petersson inner product may be unfolded to an integral over a vertical strip. For the higher-order inner product the strip is refolded and detailed knowledge of $E_{\mathfrak{a}}^{m, n}(z, s)$ is required.

We view the ideas developed in this article as establishing fundamental results in the theory of higherorder non-holomorphic Eisenstein series. Numerous other authors have encountered higher-order modular forms in their research, and we mention briefly three applications that have appeared. Firstly, in [7], [8] Goldfeld defined the series $E_{\mathfrak{a}}^{m, n}$ for the first time and shows their utility in studying the distribution of modular symbols. Detailed results of this type are required to provide an approach to the $A B C$-conjecture via Goldfeld's conjectures on periods and modular symbols, as described in [6], [9]. Secondly, in [19], [20]. Petridis and Risager prove that, under a certain ordering and normalization, modular symbols follow a Gaussian distribution. Finally, in [5] it is shown that applying the Hecke operators to the residues of poles of $E_{\mathfrak{a}}^{1,0}$ on the critical line $\operatorname{Re}(s)=1 / 2$ yields an identity that can be used to construct an orthonormal basis for a certain space of Maass forms.

\section{Statement of results}

Let $\mathcal{E}^{m, n}=\left(E_{\mathfrak{a}}^{m, n}\right)$ be the $r \times 1$ column vector of Eisenstein series, where we index over all $r$ inequivalent cusps $\mathfrak{a}$ of $\Gamma$. Let $\mathcal{I}_{m, n}$ denote all pairs $\left(i_{1}, i_{2}\right)$ for $0 \leqslant i_{1} \leqslant m$ and $0 \leqslant i_{2} \leqslant n$, and with the ordering which has $\left(i_{1}, i_{2}\right)<\left(j_{1}, j_{2}\right)$ when $i_{1}<j_{1}$ or when $i_{1}=j_{1}$ and $i_{2}<j_{2}$. Let $N=r(m+1)(n+1)$ and define the $N \times 1$ column vector

$$
\mathbb{E}^{m, n}={ }^{t}\left({ }^{t} \mathcal{E}^{m-i_{1}, n-i_{2}}\right)_{\left(i_{1}, i_{2}\right) \in \mathcal{I}_{m, n}} .
$$

For example, for a group $\Gamma$ with $r=3$ inequivalent cusps, we have when $(m, n)=(2,1)$,

$$
\begin{aligned}
\mathbb{E}^{2,1} & ={ }^{t}\left({ }^{t} \mathcal{E}^{2,1},{ }^{t} \mathcal{E}^{2,0},{ }^{t} \mathcal{E}^{1,1},{ }^{t} \mathcal{E}^{1,0},{ }^{t} \mathcal{E}^{0,1},{ }^{t} \mathcal{E}^{0,0}\right), \\
& ={ }^{t}\left(E_{\mathfrak{a}}^{2,1}, E_{\mathfrak{b}}^{2,1}, E_{\mathfrak{c}}^{2,1}, E_{\mathfrak{a}}^{2,0}, E_{\mathfrak{b}}^{2,0}, E_{\mathfrak{c}}^{2,0}, \cdots, E_{\mathfrak{a}}^{0,0}, E_{\mathfrak{b}}^{0,0}, E_{\mathfrak{c}}^{0,0}\right) .
\end{aligned}
$$

In this notation, we now state our first main result.

Theorem 2.1. There exists an explicit representation $\pi^{m, n}$ of $\Gamma$ into $\operatorname{Uni}(N, \mathbf{C})$, the space of $N \times N$ unipotent matrices, such that for all $\gamma \in \Gamma$ and $\operatorname{Re}(s)>1$, we have

$$
\mathbb{E}^{m, n}(\gamma z, s)=\pi^{m, n}(\gamma) \mathbb{E}^{m, n}(z, s) .
$$

Furthermore

$$
\Delta \mathbb{E}^{m, n}(z, s)=s(1-s) \mathbb{E}^{m, n}(z, s),
$$

where $\Delta$ denotes the usual hyperbolic Laplacian $-4 y^{2}(d / d z)(d / d \bar{z})$.

One can restate Theorem 2.1 as follows. There exists a unipotent representation $\pi^{m, n}$ of $\Gamma$ and associated vector bundle $\mathcal{V}_{m, n}$ over $\Gamma \backslash \mathbf{H}$ such that for $\operatorname{Re}(s)>1$, the vector $\mathbb{E}^{m, n}$ is a $C^{\infty}$ section of $\mathcal{V}_{m, n}$; in addition, $\mathbb{E}^{m, n}$ is an eigenvector under the action by the Laplacian $\Delta$ with eigenvalue $s(1-s)$. If $(m, n)=(0,0)$, then Theorem 2.1 reduces to well-known properties of the classical non-holomorphic Eisenstein series. For all other pairs $(m, n)$, Theorem 2.1 encodes the multiplicative and additive behavior of the higher-order non-holomorphic Eisenstein series when acted upon by the group $\Gamma$ and the Laplacian $\Delta$. 
Theorem 2.1 shows that $\mathbb{E}$ is a vector-valued automorphic form in the sense of Knopp and Mason [15], [16]. Non-holomorphic Eisenstein series associated to monomial representations (matrices with one nonzero entry in each row and column) are studied in [23]. These series arise naturally when attempting to extend the Rankin-Selberg method, and Taylor in [23] has shown the meromorphic continuation of such series.

Our next result determines the meromorphic continuation and functional equation for the vector $\mathbb{E}^{m, n}$. Recall that $\langle\gamma, f\rangle=\langle\gamma, g\rangle=0$ for $\gamma \in \Gamma_{\mathfrak{b}}$, since $f$ and $g$ are cusp forms, so then, for any cusps $\mathfrak{a}$ and $\mathfrak{b}$, we have that $E_{\mathfrak{a}}^{m, n}\left(\sigma_{\mathfrak{b}}(z+1), s\right)=E_{\mathfrak{a}}^{m, n}\left(\sigma_{\mathfrak{b}}(z), s\right)$. We conclude that the Eisenstein series $E_{\mathfrak{a}}^{m, n}$ admits a Fourier expansion at the cusp $\mathfrak{b}$ which can be shown to be

$$
E_{\mathfrak{a}}^{m, n}\left(\sigma_{\mathfrak{b}} z, s\right)=\delta_{0,0}^{m, n} \cdot \delta_{\mathfrak{a} \mathfrak{b}} y^{s}+\phi_{\mathfrak{a} \mathfrak{b}}^{m, n}(s) y^{1-s}+\sum_{k \neq 0} \phi_{\mathfrak{a} \mathfrak{b}}^{m, n}(k, s) W_{s}(k z),
$$

where $z=x+i y$,

$$
W_{s}(k z)=2 \sqrt{|k| y} K_{s-1 / 2}(2 \pi|k| y) e^{2 \pi i k x}
$$

and $K_{s-1 / 2}$ is the $K$-Bessel function. The factor $\delta_{0,0}^{m, n}$ is 1 if $(m, n)=(0,0)$ and 0 otherwise. Also $\delta_{\mathfrak{a b}}$ is 1 if $\mathfrak{a}=\mathfrak{b}$ and 0 otherwise. Equation (2.4) reveals an important property of $E^{m, n}$ : For $(m, n) \neq(0,0)$ and $\operatorname{Re}(s)>1, E^{m, n}$ has polynomial decay at every cusp. In essence, this holds because of the definition (1.1) and since $\left\langle\mathrm{I}_{2}, f\right\rangle=\left\langle\mathrm{I}_{2}, g\right\rangle=0$ where we use $\mathrm{I}_{k}$ to denote the $k \times k$ identity matrix.

For $(m, n)=(0,0)$, the expansion (2.4) is proved in [11, (3.20)] and that proof generalizes to the case $(m, n)=(1,0)$ in [17] and easily to all other $(m, n)$. Furthermore, the coefficients $\phi_{\mathfrak{a} \mathfrak{b}}^{m, n}(k, s)$ can be identified in terms of Kloosterman sums twisted by modular symbols.

For fixed $i$ and $j$, let us assemble the functions $\phi_{\mathfrak{a} \mathfrak{b}}^{i, j}(s)$ in the constant term (in $x$ ) of (2.4) into an $r \times r$ matrix

$$
\Phi^{i, j}(s)=\left(\phi_{\mathfrak{a} \mathfrak{b}}^{i, j}(s)\right) .
$$

We now construct $\Phi_{\mathbb{E}}^{m, n}$, an $N \times N$ matrix from the $r \times r$ matrices $\Phi^{i, j}$ by setting

$$
\Phi_{\mathbb{E}}^{m, n}=\left(\left(\begin{array}{c}
m-i_{1} \\
j_{1}-i_{1}
\end{array}\right)\left(\begin{array}{c}
n-i_{2} \\
j_{2}-i_{2}
\end{array}\right) \Phi^{j_{1}-i_{1}, j_{2}-i_{2}}\right)_{\left(\left(i_{1}, i_{2}\right),\left(j_{1}, j_{2}\right)\right) \in \mathcal{I}_{m, n} \times \mathcal{I}_{m, n}}
$$

where we understand that $\Phi^{u, v}=0$ if $u$ or $v$ is negative. Note that we are following standard conventions for binomial coefficients which set $\left(\begin{array}{l}0 \\ 0\end{array}\right)=1$ and $\left(\begin{array}{l}n \\ k\end{array}\right)=0$ if $k<0$ or $k>n$.

Theorem 2.2. The vector of Eisenstein series $\mathbb{E}^{m, n}(z, s)$ admits a meromorphic continuation to all $s \in \mathbf{C}$ and satisfies the functional equation

$$
\mathbb{E}^{m, n}(z, 1-s)=\Phi_{\mathbb{E}}^{m, n}(1-s) \mathbb{E}^{m, n}(z, s) .
$$

In addition, the matrix $\Phi_{\mathbb{E}}^{m, n}$ satisfies the identity

$$
\Phi_{\mathbb{E}}^{m, n}(1-s) \Phi_{\mathbb{E}}^{m, n}(s)=\mathrm{I}_{N} .
$$

In the case when $(m, n)=(0,0)$, Theorem 2.2 is a classical result of Selberg [21]. When $(m, n)=(1,0)$, the proof of Theorem 2.2 appears in [17] where Selberg's original methods in the case $(m, n)=(0,0)$ were modified, taking into account that $\mathcal{E}^{1,0}$ is no longer $\Gamma$-invariant. However, the notation in [17] does not reveal the structure implied by the notation in Theorem 2.1 or Theorem 2.2, which is one important part of the point of view taken here. Without the notation of Theorem 2.2, the functional equations (2.5) and (2.6) can be shown to be equivalent to the formulas

$$
\begin{aligned}
\sum_{\substack{0 \leqslant i \leqslant m \\
0 \leqslant j \leqslant n}}\left(\begin{array}{c}
m \\
i
\end{array}\right)\left(\begin{array}{c}
n \\
j
\end{array}\right) \Phi^{i, j}(1-s) \mathcal{E}^{m-i, n-j}(z, s) & =\mathcal{E}^{m, n}(z, 1-s), \\
\sum_{\substack{0 \leqslant i \leqslant m \\
0 \leqslant j \leqslant n}}\left(\begin{array}{c}
m \\
i
\end{array}\right)\left(\begin{array}{c}
n \\
j
\end{array}\right) \Phi^{i, j}(1-s) \Phi^{m-i, n-j}(s) & = \begin{cases}I_{r} & \text { if } m=n=0, \\
0 & \text { otherwise }\end{cases}
\end{aligned}
$$


These results should be compared with those of Risager in [20, Chapter 4] where a functional equation is found with matrix entries involving perturbation coefficients. These coefficients are also expressed there in terms of certain Dirichlet series.

The next result is an example of the growth properties of $E_{\mathfrak{a}}^{m, n}$ which we prove in section 6 . We shall use the notation $s=\sigma+i t$ throughout the paper.

Theorem 2.3. For each compact set $\mathbf{S} \subset \mathbf{C}$ and all integers $m, n \geqslant 0$, there exists a holomorphic function $\xi_{\mathbf{S}}^{m, n}(s)$ such that for all $s$ in $\mathbf{S}$ and all $k \neq 0$, we have

$$
\xi_{\mathbf{S}}^{m, n}(s) \phi_{\mathfrak{a} \mathfrak{b}}^{m, n}(k, s) \ll\left(\log ^{m+n}|k|+1\right)\left(|k|^{\sigma}+|k|^{1-\sigma}\right) .
$$

The implied constant depends solely on $f, g$ and $\Gamma$.

Finally, we note that the most general form of our theory of higher-order non-holomorphic Eisenstein series includes more that two modular symbols. As shown in [10], for example, the space of all homomorphisms from $\Gamma$ to $\mathbf{C}$ that are zero on parabolic elements is $2 g$ dimensional, where $g$ is the genus of $\Gamma \backslash \mathbf{H}$, and is generated by modular symbols and their conjugates. If $h_{i}$ is a basis of such homomorphisms, then we may consider the general series

$$
E_{\mathfrak{a}}^{m_{1}, m_{2}, \cdots, m_{2 g}}\left(z, s ; h_{1}, h_{2}, \cdots, h_{2 g}, \chi\right)=\sum_{\gamma \in \Gamma_{\mathfrak{a}} \backslash \Gamma} \chi(\gamma) h_{1}(\gamma)^{m_{1}} h_{2}(\gamma)^{m_{2}} \cdots h_{2 g}(\gamma)^{m_{2 g}} \operatorname{Im}\left(\sigma_{\mathfrak{a}}{ }^{-1} \gamma z\right)^{s} .
$$

As the reader may verify, the methods in this article apply with very few changes to the series (2.10). Homomorphisms that are not zero on all parabolic elements can also be used in (2.10); see [10] for some initial results on these series.

\section{Some preliminary results}

\subsection{Higher-order forms}

Let us set $\mathcal{A}^{0}(\Gamma)=\{0\}$ and, for $n \geqslant 1$, define $\mathcal{A}^{n}(\Gamma)$ to be the space of smooth functions $\psi: \mathbf{H} \rightarrow \mathbf{C}$ such that

$$
\psi(\gamma z)-\psi(z) \in \mathcal{A}^{n-1}(\Gamma) \text { for all } \gamma \in \Gamma .
$$

If $n=1$, then $\mathcal{A}^{1}(\Gamma)$ is the well-known space of automorphic functions. We call the elements of $\mathcal{A}^{n}(\Gamma) n$ thorder automorphic forms. In this general setting, $n$ th-order automorphic forms were first described in [14] and [1].

More completely, let $j(\gamma, z)$ be the usual automorphy factor associated to automorphic forms. For $\gamma \in \Gamma$, character $\chi$ of $\Gamma$, and integer $k \in \mathbf{Z}$, the slash operator is defined on a function $f$ by

$$
\left(\left.f\right|_{k, \chi} \gamma\right)(z)=\frac{f(\gamma z)}{j(\gamma, z)^{k} \chi(\gamma)}
$$

Recursively we define $\mathcal{A}_{k}^{n}(\Gamma, \chi)$ to be the space of all smooth functions such that

$$
\left.\psi\right|_{k, \chi} \gamma-\psi \in \mathcal{A}_{k}^{n-1}(\Gamma, \chi) \text { for all } \gamma \in \Gamma,
$$

where, as before, we set $\mathcal{A}_{k}^{0}(\Gamma, \chi)=\{0\}$. In words, $\mathcal{A}_{k}^{n}(\Gamma, \chi)$ is the space of weight $k, n$ th-order automorphic forms which are twisted by the character $\chi$. Observe that if we set $\xi_{\gamma}=\left.\psi\right|_{k, \chi} \gamma-\psi$ then $\xi_{\gamma}$, as the notation indicates, may depend on $\gamma$, though not arbitrarily: we necessarily have that $\xi_{\gamma \tau}=\left.\xi_{\gamma}\right|_{k, \chi} \tau+\xi_{\tau}$ for all $\gamma, \tau \in \Gamma$.

Lemma 3.1. For any $0 \leqslant n \leqslant m$ we have $\mathcal{A}_{k}^{n}(\Gamma, \chi) \subseteq \mathcal{A}_{k}^{m}(\Gamma, \chi)$.

Proof. We use induction on $n$. Trivially, we have that $\mathcal{A}_{k}^{0}(\Gamma, \chi) \subseteq \mathcal{A}_{k}^{m}(\Gamma, \chi)$ for any $m \geqslant 0$. This is the base case. For the inductive step:

$$
\begin{aligned}
\psi \in \mathcal{A}_{k}^{n}(\Gamma, \chi) & \left.\Longrightarrow \psi\right|_{k, \chi} \gamma-\psi \in \mathcal{A}_{k}^{n-1}(\Gamma, \chi) \\
& \left.\Longrightarrow \psi\right|_{k, \chi} \gamma-\psi \in \mathcal{A}_{k}^{m-1}(\Gamma, \chi), m \geqslant n \text { (by induction) } \\
& \Longrightarrow \psi \in \mathcal{A}_{k}^{m}(\Gamma, \chi) .
\end{aligned}
$$

This proves the claim. 
Proposition 3.2. For any $n, m \geqslant 1$, if $f \in \mathcal{A}_{k}^{n}\left(\Gamma, \chi_{1}\right)$ and $g \in \mathcal{A}_{l}^{m}\left(\Gamma, \chi_{2}\right)$ then

$$
f \cdot g \in \mathcal{A}_{k+l}^{n+m-1}\left(\Gamma, \chi_{1} \chi_{2}\right) .
$$

Proof. We use induction on $n+m$. Observe that

$$
\left.(f \cdot g)\right|_{k+l, \chi_{1} \chi_{2}} \gamma=\left(\left.f\right|_{k, \chi_{1}} \gamma\right)\left(\left.g\right|_{l, \chi_{2}} \gamma\right)=\left(f+f_{1}\right)\left(g+g_{1}\right)
$$

for some $f_{1} \in \mathcal{A}_{k}^{n-1}\left(\Gamma, \chi_{1}\right)$ and $g_{1} \in \mathcal{A}_{l}^{m-1}\left(\Gamma, \chi_{2}\right)$. Hence

$$
\left.(f \cdot g)\right|_{k+l, \chi_{1} \chi_{2}} \gamma-f \cdot g=f_{1} \cdot g+f \cdot g_{1}+f_{1} \cdot g_{1} .
$$

By induction and Lemma 3.1, we have that both $f_{1} \cdot g$ and $f \cdot g_{1}$ are elements of $\mathcal{A}_{k+l}^{n+m-2}\left(\Gamma, \chi_{1} \chi_{2}\right)$, and $f_{1} \cdot g_{1} \in \mathcal{A}_{k+l}^{n+m-3}\left(\Gamma, \chi_{1} \chi_{2}\right) \subseteq \mathcal{A}_{k+l}^{n+m-2}\left(\Gamma, \chi_{1} \chi_{2}\right)$. Therefore, $\left.f \cdot g\right|_{k+l, \chi_{1} \chi_{2}} \gamma-f \cdot g \in \mathcal{A}_{k+l}^{n+m-2}\left(\Gamma, \chi_{1} \chi_{2}\right)$ which implies that $f \cdot g \in \mathcal{A}_{k+l}^{n+m-1}\left(\Gamma, \chi_{1} \chi_{2}\right)$ as required. This proves the induction step.

It remains to prove the first step in the induction, namely when $(n, m)=(1,1)$. This follows immediately from the observation that the product of two classical automorphic forms of weight $k$ and $l$ is an automorphic form of weight $k+l$. With all this, the proof of the proposition is complete.

Remark: We can interpret Lemma 3.1 and Proposition 3.2 as proving that the space of all higher-order automorphic forms has a graded ring structure. In [3] it is shown that the theory of Rankin-Cohen brackets applies and gives the set of higher-order forms a canonical RC structure. It is natural to ask if, under either structure, the graded ring of higher-order holomorphic automorphic forms is finitely generated.

\subsection{Higher-order Eisenstein series}

We will show that $E_{\mathfrak{a}}^{m, n} \in \mathcal{A}_{0}^{m+n+1}(\Gamma, \chi)$ in Lemma 4.2. In this section, we establish some more basic properties.

To see that the terms in (1.1) are well defined we note that $\langle\gamma, f\rangle=0$ whenever $\gamma \in \Gamma_{\mathfrak{a}}$. Therefore, the modular symbols are well-defined functions on the cosets $\Gamma_{\mathfrak{a}} \backslash \Gamma$. Similarly, we also require that $\chi(\gamma)=1$ for all $\gamma \in \Gamma_{\mathfrak{a}}$ and for convergence the character must satisfy $|\chi|=1$.

The size of an automorphic form in cuspidal zones is of great importance. Following [11], we will measure the growth of automorphic forms using the invariant height function defined by

$$
y_{\Gamma}(z)=\max _{\mathfrak{a}} \max _{\gamma \in \Gamma}\left(\operatorname{Im}\left(\sigma_{\mathfrak{a}}{ }^{-1} \gamma z\right)\right) .
$$

Suppose that $\psi$ is a continuous function on $\mathbf{H}$ such that $|\psi|$ is $\Gamma$-invariant, and let $B$ denote a continuous positive function on $[0, \infty)$. Then it is easy to verify that the following conditions are equivalent:

(i) $\psi\left(\sigma_{\mathfrak{a}} z\right) \ll B(y)$ as $y \rightarrow \infty$ for all cusps $\mathfrak{a}$,

(ii) $\psi(z) \ll B\left(y_{\Gamma}(z)\right)$.

More generally, for continuous functions $\xi$ on $\mathbf{H}$ which are not necessarily automorphic, it is more convenient to fix a fundamental domain, $\mathbf{F}$, and examine their growth on $\mathbf{F}$. Let $\mathcal{P}_{Y} \subset \mathbf{H}$ denote the strip with $|x| \leqslant 1 / 2$ and $y \geqslant Y$. We choose $\mathbf{F}$ so that it contains the cuspidal zones $\sigma_{\mathfrak{a}} \mathcal{P}_{Y}$ for all $\mathfrak{a}$ and $Y$ large enough (see [11, Section 2.2]). For $z \in \mathbf{F}$ we define

$$
y_{\mathbf{F}}(z)=\max _{\mathfrak{a}}\left(\operatorname{Im}\left(\sigma_{\mathfrak{a}}{ }^{-1} z\right)\right)
$$

to be the domain height function; with this definition, we assert that the following statements are equivalent

(i) $\xi\left(\sigma_{\mathfrak{a}} z\right) \ll B(y)$ as $y \rightarrow \infty$ for all cusps $\mathfrak{a}$ and all $|x| \leqslant 1 / 2$,

(ii) $\xi(z) \ll B\left(y_{\mathbf{F}}(z)\right)$ for all $z \in \mathbf{F}$. 
Also we observe the relation

$$
y_{\mathbf{F}}(z) \leqslant y_{\Gamma}(z) .
$$

Further required properties of the invariant height are proved in Appendix A.

Proposition 3.3. For $m, n \geqslant 0$ and $(m, n) \neq(0,0)$ the series (1.1 defining $E_{\mathfrak{a}}^{m, n}(z, s)$ converges to a smooth function of $z \in \mathbf{H}$ and holomorphic function of $s \in \mathbf{C}$ provided $\operatorname{Re}(s)>1$. The convergence is absolute for any $s \in \mathbf{C}$ with $\operatorname{Re}(s)>1$ and uniform in the region $\operatorname{Re}(s) \geqslant 1+\delta$ for any $\delta>0$. Furthermore, for $\operatorname{Re}(s)=\sigma>1$, the function $E_{\mathfrak{a}}^{m, n}(z, s)$ satisfies the growth condition

$$
E_{\mathfrak{a}}^{m, n}(z, s) \ll y_{\mathbf{F}}(z)^{1-\sigma+\varepsilon}
$$

for any $\varepsilon>0$ and all $z \in \mathbf{F}$. The implied constant depends only on $\varepsilon, \sigma, m, n, f, g$ and $\Gamma$.

Proof. Set

$$
F_{\mathfrak{a}}(z)=\int_{\mathfrak{a}}^{z} f(w) d w, G_{\mathfrak{a}}(z)=\int_{\mathfrak{a}}^{z} g(w) d w .
$$

First we note that, for any two (possibly equal) cusps $\mathfrak{a}$ and $\mathfrak{b}$ and for all $z \in \mathbf{H}$,

$$
F_{\mathfrak{a}}\left(\sigma_{\mathfrak{b}} z\right) \ll|\log y|+1
$$

with the same bound for $G_{\mathfrak{a}}$, where the implicit constant in (3.3) depends solely on $f$ and $\Gamma$. To prove (3.3), observe that the integral of $f(z)$ from $z$ to $z+1$ is zero, and that $f(z) \ll 1 / y$ (see [4, Lemma 3]). It also follows from (3.3) that

$$
F_{\mathfrak{a}}(z) \ll \log \left(y_{\mathbf{F}}(z)+e\right) .
$$

We shall need some elementary inequalities in the sequel:

$$
\begin{aligned}
|\log y| & <\left(y^{\epsilon}+y^{-\epsilon}\right) / \epsilon, \\
|\log y|+1 & <3 \log (y+1 / y), \\
(y+1)^{r} & <2^{r}\left(y^{r}+1\right), \\
(y+1 / y)^{r} & <2^{r}\left(y^{r}+y^{-r}\right)
\end{aligned}
$$

for all $y, \epsilon, r>0$.

From (3.3) and (3.5), we deduce that

$$
\begin{aligned}
\langle\gamma, f\rangle & =F_{\mathfrak{a}}(\gamma z)-F_{\mathfrak{a}}(z) \\
& \ll\left|\log \operatorname{Im}\left(\sigma_{\mathfrak{b}}{ }^{-1} \gamma z\right)\right|+\left|\log \operatorname{Im}\left({\sigma_{\mathfrak{b}}}^{-1} z\right)\right|+1 \\
& \ll \operatorname{Im}\left(\sigma_{\mathfrak{b}}{ }^{-1} \gamma z\right)^{\varepsilon}+\operatorname{Im}\left({\sigma_{\mathfrak{b}}}^{-1} \gamma z\right)^{-\varepsilon}+\operatorname{Im}\left(\sigma_{\mathfrak{b}}{ }^{-1} z\right)^{\varepsilon}+\operatorname{Im}\left({\sigma_{\mathfrak{b}}}^{-1} z\right)^{-\varepsilon} .
\end{aligned}
$$

Hence, for any $\varepsilon>0$, any $z \in \mathbf{H}$ and any cusp $\mathfrak{a}$,

$$
\langle\gamma, f\rangle^{m}\langle\gamma, g\rangle^{n} \ll \operatorname{Im}\left({\sigma_{\mathfrak{a}}}^{-1} \gamma z\right)^{\varepsilon}+\operatorname{Im}\left({\sigma_{\mathfrak{a}}}^{-1} \gamma z\right)^{-\varepsilon}+\operatorname{Im}\left({\sigma_{\mathfrak{a}}}^{-1} z\right)^{\varepsilon}+\operatorname{Im}\left({\sigma_{\mathfrak{a}}}^{-1} z\right)^{-\varepsilon}
$$

with an implied constant depending on $f, g, \Gamma, m, n$ and $\varepsilon$. Also for any cusp $\mathfrak{a}$ and any $z \in \mathbf{F}$

$$
\operatorname{Im}\left({\sigma_{\mathfrak{a}}}^{-1} z\right) \leqslant y_{\mathbf{F}}(z)
$$

by definition. It is well-known that the classical non-holomorphic Eisenstein series, meaning (1.1) for $(m, n)=(0,0)$, converges for $\operatorname{Re}(s)>1$ (uniformly for $\operatorname{Re}(s) \geqslant 1+\delta)$ and satisfies the bound

$$
E_{\mathfrak{a}}(z, s) \ll y_{\Gamma}(z)^{\sigma} ;
$$

see, for example, [11, Corollary 3.5]. Going further, we have that

$$
E_{\mathfrak{a}}(z, s)-\operatorname{Im}\left(\sigma_{\mathfrak{a}}{ }^{-1} z\right)^{s} \ll y_{\mathbf{F}}(z)^{1-\sigma},
$$

for $z \in \mathbf{F}$; for the sake of completeness, we will prove (3.12) in detail in Lemma A.3. Since $\left\langle\mathrm{I}_{2}, f\right\rangle=$ $\left\langle\mathrm{I}_{2}, g\right\rangle=0$, the term in (1.1) corresponding to the identity coset vanishes for $(m, n) \neq(0,0)$. With all this, we can substitute the bound (3.9) into the definition (1.1) and use (3.10) and (3.12) to complete the proof of the proposition. 


\section{Unipotent higher-order vector bundles}

\subsection{Automorphy factors}

For any $f \in S_{2}(\Gamma)$ it is an easy consequence of elementary complex analysis that, for all $\gamma_{1}, \gamma_{2} \in \Gamma$,

$$
\left\langle\gamma_{1} \gamma_{2}, f\right\rangle=\left\langle\gamma_{1}, f\right\rangle+\left\langle\gamma_{2}, f\right\rangle
$$

This is the basis for the following computation.

Lemma 4.1. For any $\tau \in \Gamma$, let

$$
S_{i, j}(\tau)=\bar{\chi}(\tau)(-\langle\tau, f\rangle)^{i}(-\overline{\langle\tau, g\rangle})^{j} .
$$

For $\operatorname{Re}(s)>1$, the Eisenstein series $E_{\mathfrak{a}}^{m, n}$ obeys the transformation rule

$$
E_{\mathfrak{a}}^{m, n}(\tau z, s)=\sum_{i=0}^{m} \sum_{j=0}^{n}\left(\begin{array}{c}
m \\
i
\end{array}\right)\left(\begin{array}{c}
n \\
j
\end{array}\right) S_{m-i, n-j}(\tau) E_{\mathfrak{a}}^{i, j}(z, s) .
$$

Proof. Combining (1.1) and (4.1), we get

$$
\begin{aligned}
E_{\mathfrak{a}}^{m, n}(\tau z, s) & =\sum_{\gamma \in \Gamma_{\mathfrak{a}} \backslash \Gamma} \chi(\gamma)\langle\gamma, f\rangle^{m} \overline{\langle\gamma, g\rangle}^{n} \operatorname{Im}\left({\sigma_{\mathfrak{a}}}^{-1} \gamma \tau z\right)^{s} \\
& =\sum_{\gamma \in \Gamma_{\mathfrak{a}} \backslash \Gamma} \chi\left(\gamma \tau^{-1}\right)\left\langle\gamma \tau^{-1}, f\right\rangle^{m}{\overline{\left\langle\gamma \tau^{-1}, g\right\rangle}}^{n} \operatorname{Im}\left(\sigma_{\mathfrak{a}}{ }^{-1} \gamma z\right)^{s} \\
& =\sum_{\gamma \in \Gamma_{\mathfrak{a}} \backslash \Gamma} \chi(\gamma) \bar{\chi}(\tau)(\langle\gamma, f\rangle-\langle\tau, f\rangle)^{m} \cdot(\overline{\langle\gamma, g\rangle}-\overline{\langle\tau, g\rangle})^{n} \operatorname{Im}\left(\sigma_{\mathfrak{a}}{ }^{-1} \gamma z\right)^{s} .
\end{aligned}
$$

The result now follows by expanding the terms $(\langle\gamma, f\rangle-\langle\tau, f\rangle)^{m}$ and $(\overline{\langle\gamma, g\rangle}-\overline{\langle\tau, g\rangle})^{n}$.

Lemma 4.2. For $m, n \geqslant 0$ we have $E_{\mathfrak{a}}^{m, n} \in \mathcal{A}_{0}^{m+n+1}(\Gamma, \chi)$.

Proof. Using (4.2) we observe that $\left.E_{\mathfrak{a}}^{0,0}\right|_{0, \chi} \gamma-E_{\mathfrak{a}}^{0,0}=0$ and $E_{\mathfrak{a}}^{0,0} \in \mathcal{A}_{0}^{1}(\Gamma, \chi)$. Now, using induction on $m+n$, we have by (4.2) that $\left.E_{\mathfrak{a}}^{m, n}\right|_{0, \chi} \gamma-E_{\mathfrak{a}}^{m, n}$ is a linear combination of lower order Eisenstein series that are in $\mathcal{A}_{0}^{m+n}(\Gamma, \chi)$. The lemma follows.

We now complete the proof of Theorem 2.1.

Proof. Directly from (4.2) we obtain the relation

$$
\mathcal{E}^{m, n}(\gamma z, s)=\sum_{i=0}^{m} \sum_{j=0}^{n}\left(\begin{array}{c}
m \\
i
\end{array}\right)\left(\begin{array}{c}
n \\
j
\end{array}\right) S_{m-i, n-j}(\gamma) \mathcal{E}^{i, j}(z, s)
$$

for the $r \times 1$ vector of Eisenstein series $\mathcal{E}^{m, n}$. Construct the $N \times N$ matrix

$$
\pi^{m, n}(\gamma)=\left(\left(\begin{array}{c}
m-i_{1} \\
m-j_{1}
\end{array}\right)\left(\begin{array}{c}
n-i_{2} \\
n-j_{2}
\end{array}\right) S_{j_{1}-i_{1}, j_{2}-i_{2}}(\gamma) \mathrm{I}_{r}\right)_{\left(\left(i_{1}, i_{2}\right),\left(j_{1}, j_{2}\right)\right) \in \mathcal{I}_{m, n} \times \mathcal{I}_{m, n}} .
$$

If we replace $(m, n)$ in (4.3) by $(u, v)$, say, then it is elementary to verify that (4.3) can be combined for all $(u, v) \leqslant(m, n)$ in the single equation

$$
\mathbb{E}^{m, n}(\gamma z, s)=\pi^{m, n}(\gamma) \mathbb{E}^{m, n}(z, s),
$$

which holds for all $\gamma \in \Gamma$, proving (2.2). As for (2.3), this relation following directly from (1.1) when differentiating term by term, using that $\Delta y^{s}=s(1-s) y^{s}$ and the fact that $\Delta$ is $\mathrm{SL}_{2}(\mathbf{R})$ invariant.

The matrix $\pi^{m, n}(\tau)$ is easily seen to be upper triangular with ones on the diagonal, hence it is unipotent. Upon replacing $z$ by $\gamma z$ and repeating (4.5), we obtain the relation $\pi^{m, n}(\tau \gamma)=\pi^{m, n}(\tau) \pi(\gamma)$. Therefore $\pi^{m, n}$ is a representation of $\Gamma$ into the set of $N \times N$ unipotent matrices with $N=r(m+1)(n+1)$. Consequently, the representation $\pi^{m, n}$ can be used to define a rank $N$ unipotent vector bundle $\mathcal{V}^{m, n}$ over $\Gamma \backslash \mathbf{H}$, and (2.2) is simply the statement that $\mathbb{E}^{m, n}(z, s)$ is a section of $\mathcal{V}^{m, n}$.

With all this, the proof of Theorem 2.1 is complete. 


\subsection{An example}

A simple case of Theorem 2.1 occurs when $(m, n)=(2,0)$, for an arbitrary number of cusps $r$. In this instance, we see from (4.4) that

$$
\pi^{2,0}(\gamma)=\bar{\chi}(\gamma)\left(\begin{array}{ccc}
\mathrm{I}_{r} & -2\langle\gamma, f\rangle \cdot \mathrm{I}_{r} & \langle\gamma, f\rangle^{2} \cdot \mathrm{I}_{r} \\
& \mathrm{I}_{r} & -\langle\gamma, f\rangle \cdot \mathrm{I}_{r} \\
& \mathrm{I}_{r}
\end{array}\right)
$$

and (4.5) says $\mathbb{E}^{2,0}(\gamma z, s)=\pi^{2,0}(\gamma) \mathbb{E}^{2,0}(z, s)$ which encodes the three identities

$$
\begin{aligned}
& \mathcal{E}^{2,0}(\gamma z, s)=\bar{\chi}(\gamma)\left(\mathcal{E}^{2,0}(z, s)-2\langle\gamma, f\rangle \mathcal{E}^{1,0}(z, s)+\langle\gamma, f\rangle^{2} \mathcal{E}^{0,0}(z, s)\right) \\
& \mathcal{E}^{1,0}(\gamma z, s)=\bar{\chi}(\gamma)\left(\mathcal{E}^{1,0}(z, s)-\langle\gamma, f\rangle \mathcal{E}^{0,0}(z, s)\right) \\
& \mathcal{E}^{0,0}(\gamma z, s)=\bar{\chi}(\gamma) \mathcal{E}^{0,0}(z, s)
\end{aligned}
$$

\section{Constructing an integral equation for $E_{\mathfrak{a}}^{m, n}$}

\subsection{Bounds for $E_{\mathfrak{a}}^{m, n}$ on $\mathbf{H}$}

We need bounds for $E_{\mathfrak{a}}^{m, n}$ on the upper half plane $\mathbf{H}$. The fact that it is not $\Gamma$-invariant makes this hard to do directly, so we introduce a related automorphic series.

For non-negative integers $m$ and $n$, we define, using the notation in (3.2), the function

$$
Q_{\mathfrak{a}}^{m, n}(z, s)=\sum_{\gamma \in \Gamma_{\mathfrak{a}} \backslash \Gamma} \chi(\gamma) F_{\mathfrak{a}}(\gamma z)^{m}{\overline{G_{\mathfrak{a}}(\gamma z)}}^{n} \operatorname{Im}\left({\sigma_{\mathfrak{a}}}^{-1} \gamma z\right)^{s}
$$

which will naturally be automorphic wherever it converges. Observe that when $m=n=0$ the series (5.1) is just the usual Eisenstein series $E_{\mathfrak{a}}(z, s)$. To see the connection between $E_{\mathfrak{a}}^{m, n}$ and $Q_{\mathfrak{a}}^{m, n}$ use the identity $F_{\mathfrak{a}}(\gamma z)-F_{\mathfrak{a}}(z)=\langle\gamma, f\rangle$ and the binomial theorem to obtain

$$
\begin{aligned}
Q_{\mathfrak{a}}^{m, n}(z, s) & =\sum_{i, j}\left(\begin{array}{c}
m \\
i
\end{array}\right)\left(\begin{array}{c}
n \\
j
\end{array}\right) F_{\mathfrak{a}}(z)^{m-i}{\overline{G_{\mathfrak{a}}(z)}}^{n-j} E_{\mathfrak{a}}^{i, j}(z, s), \\
E_{\mathfrak{a}}^{m, n}(z, s) & =\sum_{i, j}\left(\begin{array}{c}
m \\
i
\end{array}\right)\left(\begin{array}{c}
n \\
j
\end{array}\right)\left(-F_{\mathfrak{a}}(z)\right)^{m-i}\left(-\overline{G_{\mathfrak{a}}(z)}\right)^{n-j} Q_{\mathfrak{a}}^{i, j}(z, s) .
\end{aligned}
$$

Since $E_{\mathfrak{a}}^{i, j}(z, s)$ is convergent for $\operatorname{Re}(s)>1$, so is $Q_{\mathfrak{a}}^{m, n}(z, s)$ by (5.2). Using (3.1), (3.4) and (3.11) to bound the right side of (5.2), we get

$$
Q_{\mathfrak{a}}^{m, n}(z, s) \ll \log ^{m+n}\left(y_{\mathbf{F}}(z)+e\right) \cdot y_{\mathbf{F}}(z)^{\sigma}
$$

where the implied constant depends on $\epsilon, \sigma, m, n, f, g$ and $\Gamma$ alone and $z$ is restricted to $\mathbf{F}$. Since $Q_{\mathfrak{a}}^{m, n}$ is automorphic, and the right side of (5.4) is an increasing function of $y_{\mathbf{F}}$, it follows that, for all $m, n \geqslant 0$ and $z \in \mathbf{H}$,

$$
Q_{\mathfrak{a}}^{m, n}(z, s) \ll \log ^{m+n}\left(y_{\Gamma}(z)+e\right) \cdot y_{\Gamma}(z)^{\sigma} .
$$

We need the next result, which is a slightly stronger and more explicit version of [12, Lemma 7.1].

Lemma 5.1. Suppose $D$ is a function on $\mathbf{H}$ and $B$ is an increasing function on $\mathbf{R}^{+}$such that $|D(z)| \leqslant B\left(y_{\Gamma}(z)\right)$. Then there exists a constant $c_{\Gamma}>0$, depending only on $\Gamma$ and defined in (A.1), so that

$$
\left|D\left(\sigma_{\mathfrak{b}} z\right)\right| \leqslant B\left(\left(c_{\Gamma}+1 / c_{\Gamma}\right)(y+1 / y)\right)
$$

for any cusp $\mathfrak{b}$ and any $z \in \mathbf{H}$.

Proof. Using Lemma A.1, which is proved in the appendix to this paper, we have the bound

$$
\left|D\left(\sigma_{\mathfrak{b}} z\right)\right| \leqslant B\left(y_{\Gamma}\left(\sigma_{\mathfrak{b}} z\right)\right) \leqslant B\left(\left(c_{\Gamma}+1 / c_{\Gamma}\right)(y+1 / y)\right),
$$

as claimed. 
It follows from (5.5), Lemma 5.1 and (3.8) that

$$
\begin{aligned}
Q_{\mathfrak{a}}^{m, n}\left(\sigma_{\mathfrak{b}} z, s\right) & \ll \log ^{m+n}\left(\left(c_{\Gamma}+1 / c_{\Gamma}\right)(y+1 / y)+e\right) \cdot\left(\left(c_{\Gamma}+1 / c_{\Gamma}\right)(y+1 / y)\right)^{\sigma} \\
& \ll \log ^{m+n}(y+1 / y) \cdot\left(y^{\sigma}+y^{-\sigma}\right) .
\end{aligned}
$$

Substituting this bound into (5.3) then using (3.3) and (3.6), we obtain

$$
E_{\mathfrak{a}}^{m, n}\left(\sigma_{\mathfrak{b}} z, s\right) \ll \log ^{m+n}(y+1 / y) \cdot\left(y^{\sigma}+y^{-\sigma}\right) .
$$

As a result, we arrive at the following conclusion.

Lemma 5.2. For all $z$ in $\mathbf{H}, s$ in $\mathbf{C}$ with $\operatorname{Re}(s)>1$, and $m, n \geqslant 0$,

$$
E_{\mathfrak{a}}^{m, n}\left(\sigma_{\mathfrak{b}} z, s\right) \ll y^{\sigma+\epsilon}+y^{-\sigma-\epsilon}
$$

with an implied constant depending only on $\epsilon>0, \sigma, m, n, f, g$ and $\Gamma$.

\subsection{A family of Green functions}

Set

$$
u(z, w)=\frac{|z-w|^{2}}{4 \operatorname{Im} z \operatorname{Im} w} .
$$

This function $u$ is closely related to the hyperbolic distance between $z$ and $w$ and satisfies the relation $u(\sigma z, \sigma w)=u(z, w)$ for all $\sigma$ in $\mathrm{SL}_{2}(\mathbf{R})$; see $[11,(1.4)]$. We also need the Green function for the Laplacian:

$$
G_{a}(u)=\frac{1}{4 \pi} \int_{0}^{1}(t(1-t))^{a-1}(t+u)^{-a} d t
$$

for $u, a>0$. This function is discussed in [11, Section 1.7]. The Green function $G_{a}(u)$ is smooth as a function of $u$ except for a logarithmic singularity at $u=0$ which is independent of $a$. It can be shown that, for $0<b<a$,

$$
G_{a b}(u):=G_{a}(u)-G_{b}(u) \ll \frac{1}{(u+1)^{b}} .
$$

The next result is from [17, Theorem 2.3] as well as [11, Section 1.9] and is a restatement of the fact that the resolvent of the Laplacian can be expressed as an integral operator with kernel given by the above Green function.

Theorem 5.3. If $\theta(z): \mathbf{H} \rightarrow \mathbf{C}$ is an eigenfunction of $\Delta$ with eigenvalue $\lambda$ that satisfies $\theta(z) \ll y^{\sigma}+y^{-\sigma}$ for $\sigma>0$ then, when $a>\sigma+1$, then

$$
\frac{-\theta(w)}{\lambda+a(1-a)}=\int_{\mathbf{H}} G_{a}(u(w, z)) \theta(z) d \mu(z)
$$

where $d \mu(z)$ is the hyperbolic invariant measure $d x d y / y^{2}$.

Now we can exploit the fact that $E_{\mathfrak{a}}^{m, n}(z, s)$ is an eigenfunction of the Laplacian, (2.3), along with Lemma 5.2 and Theorem 5.3 to write

$$
\frac{-E_{\mathfrak{a}}^{m, n}(z, s)}{(a(1-a)-s(1-s))}=\int_{\mathbf{H}} G_{a}\left(u\left(z, z^{\prime}\right)\right) E_{\mathfrak{a}}^{m, n}\left(z^{\prime}, s\right) d \mu\left(z^{\prime}\right)
$$

for $1<\operatorname{Re}(s)<a-2$. With Lebesgue's theorems on monotone and dominated convergence and the notation in Lemma 4.1, we find

$$
\begin{aligned}
& \frac{-E_{\mathfrak{a}}^{m, n}(z, s)}{(a(1-a)-s(1-s))}=\int_{\mathbf{F}} \sum_{\gamma \in \Gamma} G_{a}\left(u\left(z, \gamma z^{\prime}\right)\right) E_{\mathfrak{a}}^{m, n}\left(\gamma z^{\prime}, s\right) d \mu\left(z^{\prime}\right) \\
& =\int_{\mathbf{F}} \sum_{\gamma \in \Gamma} G_{a}\left(u\left(z, \gamma z^{\prime}\right)\right)\left(\sum_{i=0}^{m} \sum_{j=0}^{n}\left(\begin{array}{c}
m \\
i
\end{array}\right)\left(\begin{array}{c}
n \\
j
\end{array}\right) S_{m-i, n-j}(\gamma) E_{\mathfrak{a}}^{i, j}(z, s)\right) d \mu\left(z^{\prime}\right) .
\end{aligned}
$$


Therefore, for $1<\operatorname{Re}(s)<a-2$ we get

$$
\begin{aligned}
\frac{-E_{\mathfrak{a}}^{m, n}(z, s)}{(a(1-a)-s(1-s))}= & \int_{\mathbf{F}} G_{a}^{0,0}\left(z, z^{\prime}\right) E_{\mathfrak{a}}^{m, n}\left(z^{\prime}, s\right) d \mu\left(z^{\prime}\right) \\
& +\sum_{\substack{0 \leq i \leq m \\
0 \leq i \leq m \\
i, j) \neq(m, n)}}\left(\begin{array}{c}
m \\
i
\end{array}\right)\left(\begin{array}{c}
n \\
j
\end{array}\right) \int_{\mathbf{F}} G_{a}^{m-i, n-j}\left(z, z^{\prime}\right) E_{\mathfrak{a}}^{i, j}\left(z^{\prime}, s\right) d \mu\left(z^{\prime}\right)
\end{aligned}
$$

where, for $z \not \equiv z^{\prime}(\bmod \Gamma)$, we have written

$$
\begin{aligned}
G_{a}^{i, j}\left(z, z^{\prime}\right) & =\sum_{\gamma} S_{i, j}(\gamma) G_{a}\left(u\left(z, \gamma z^{\prime}\right)\right) \\
& =\sum_{\gamma} S_{i, j}\left(\gamma^{-1}\right) G_{a}\left(u\left(\gamma z, z^{\prime}\right)\right) .
\end{aligned}
$$

The function $G_{a}^{i, j}\left(z, z^{\prime}\right)$ will inherit a logarithmic singularity at $z \equiv z^{\prime}(\bmod \Gamma)$. To eliminate the singularity, we consider the difference

$$
G_{a b}^{i, j}\left(z, z^{\prime}\right):=G_{a}^{i, j}\left(z, z^{\prime}\right)-G_{b}^{i, j}\left(z, z^{\prime}\right)=\sum_{\gamma} S_{i, j}(\gamma) G_{a b}\left(u\left(z, \gamma z^{\prime}\right)\right)
$$

for $0<b<a$. To ease the notation we write $G_{a}$ and $G_{a b}$ for $G_{a}^{0,0}$ and $G_{a b}^{0,0}$ respectively, as in [11, (5.1)]. The next Proposition follows from [11, Theorem 5.3, Lemma 5.4].

Proposition 5.4. For all real $a, b$ with $1<b<a$ we have that $G_{a b}\left(z, z^{\prime}\right): \mathbf{H} \times \mathbf{H} \rightarrow \mathbf{C}$ converges uniformly to a smooth function of $z, z^{\prime}$ which satisfies

$$
G_{a b}\left(z, z^{\prime}\right) \ll\left[y_{\Gamma}(z) y_{\Gamma}\left(z^{\prime}\right)\right]^{a}
$$

where the implied constant depends only on $a, b$ and $\Gamma$.

We need a similar result for $G_{a b}^{i, j}$.

Proposition 5.5. For all nonnegative integers $(i, j) \neq(0,0)$ and all real $a, b$ with $1<b<a$ we have that $G_{a b}^{i, j}\left(z, z^{\prime}\right)$ : $\mathbf{H} \times \mathbf{H} \rightarrow \mathbf{C}$ converges uniformly to a continuous function of $z, z^{\prime}$ which satisfies

$$
G_{a b}^{i, j}\left(z, z^{\prime}\right) \ll\left[y_{\mathbf{F}}(z) y_{\mathbf{F}}\left(z^{\prime}\right)\right]^{1-b+\epsilon}
$$

for any $\epsilon>0$ where the implied constant depends only on $\epsilon, a, b, i, j, f, g$ and $\Gamma$.

Proof. We begin by noting that

$$
\begin{aligned}
\frac{1}{\left(u\left(z, z^{\prime}\right)+1\right)^{b}} & =\frac{4^{b} \operatorname{Im}(z)^{b} \operatorname{Im}\left(z^{\prime}\right)^{b}}{\left(\left|z-z^{\prime}\right|^{2}+4 \operatorname{Im}(z) \operatorname{Im}\left(z^{\prime}\right)\right)^{b}} \\
& =\frac{4^{b} \operatorname{Im}(z)^{b} \operatorname{Im}\left(z^{\prime}\right)^{b}}{\left(\operatorname{Re}\left(z-z^{\prime}\right)^{2}+\operatorname{Im}\left(z+z^{\prime}\right)^{2}\right)^{b}} .
\end{aligned}
$$

So we have, recalling (5.6),

$$
\begin{aligned}
G_{a b}^{i, j}\left(\sigma_{\mathfrak{a}} z, z^{\prime}\right) & =\sum_{\gamma \in \Gamma} S_{i, j}(\gamma) G_{a b}\left(u\left(\sigma_{\mathfrak{a}} z, \gamma z^{\prime}\right)\right) \\
& \ll \sum_{\gamma \in \Gamma}\left|S_{i, j}(\gamma)\right| \frac{1}{\left(u\left(z, \sigma_{\mathfrak{a}}^{-1} \gamma z^{\prime}\right)+1\right)^{b}} \\
& \ll \sum_{\substack{\gamma \in \Gamma_{\mathfrak{a}} \backslash \Gamma \\
l \in \mathbf{Z}}}\left|S_{i, j}(\gamma)\right| \frac{4^{b} \operatorname{Im}(z)^{b} \operatorname{Im}\left(\sigma_{\mathfrak{a}}^{-1} \gamma z^{\prime}\right)^{b}}{\left(\operatorname{Re}\left(z-\sigma_{\mathfrak{a}}^{-1} \gamma z^{\prime}+l\right)^{2}+\operatorname{Im}\left(z+\sigma_{\mathfrak{a}}^{-1} \gamma z^{\prime}\right)^{2}\right)^{b}} .
\end{aligned}
$$

Now since

$$
\int_{-\infty}^{\infty} \frac{d x}{\left(x^{2}+y^{2}\right)^{b}}=\sqrt{\pi} \frac{\Gamma(b-1 / 2)}{\Gamma(b)} \frac{1}{y^{2 b-1}}
$$


we obtain

$$
\begin{aligned}
G_{a b}^{i, j}\left(\sigma_{\mathfrak{a}} z, z^{\prime}\right) & \ll \sum_{\gamma \in \Gamma_{\mathfrak{a}} \backslash \Gamma}\left|S_{i, j}(\gamma)\right| \frac{\operatorname{Im}(z)^{b} \operatorname{Im}\left(\sigma_{\mathfrak{a}}{ }^{-1} \gamma z^{\prime}\right)^{b}}{\operatorname{Im}\left(z+\sigma_{\mathfrak{a}}{ }^{-1} \gamma z^{\prime}\right)^{2 b-1}} \\
& \ll \operatorname{Im}(z)^{1-b} \sum_{\gamma \in \Gamma_{\mathfrak{a}} \backslash \Gamma}\left|S_{i, j}(\gamma)\right| \operatorname{Im}\left(\sigma_{\mathfrak{a}}{ }^{-1} \gamma z^{\prime}\right)^{b} .
\end{aligned}
$$

The proposition now follows as in the proof of Proposition 3.3.

\subsection{Applying Fredholm Theory}

We must carry out some modifications to equation (5.7) before we can use the next result from [11, A.4].

Theorem 5.6. (Fredholm) Assume $\int_{\mathbf{F}} d \mu\left(z^{\prime}\right)=V<\infty$ and that $K\left(z, z^{\prime}\right)$ is bounded and integrable on $\mathbf{F} \times \mathbf{F}$. Then for all $\lambda \in \mathbb{C}$ there exist $D(\lambda)$ and $D_{\lambda}\left(z, z^{\prime}\right)$ entire in $\lambda$ with the following property: If $q(z)$ is any bounded integrable function on $\mathbf{F}$ and if $h(z)$ (defined on $\mathbf{F}$ ) satisfies

$$
h(z)=q(z)+\lambda \int_{\mathbf{F}} K\left(z, z^{\prime}\right) h\left(z^{\prime}\right) d \mu\left(z^{\prime}\right)
$$

then $h(z)$ is uniquely determined and given by the formula

$$
h(z)=q(z)+\frac{\lambda}{D(\lambda)} \int_{\mathbf{F}} D_{\lambda}\left(z, z^{\prime}\right) q\left(z^{\prime}\right) d \mu\left(z^{\prime}\right)
$$

when $D(\lambda) \neq 0$.

Our situation is slightly complicated by dependence on a parameter $s$ which will be contained in a compact set $\mathbf{S} \subset \mathbb{C}$. We will set $K\left(z, z^{\prime}\right)=K_{s}\left(z, z^{\prime}\right)$ so $D(\lambda)$ and $D_{\lambda}\left(z, z^{\prime}\right)$ vary with $s$. Also $q(z)=q(z, s)$ and $\lambda=\lambda(s)$, where $q, \lambda$ and $K$ are analytic functions of $s$ on $\mathbf{S}$. If $h(z, s)$ is analytic in some neighborhood $\mathbf{S}^{\prime} \subset \mathbf{S}$ and satisfies

$$
h(z, s)=q(z, s)+\lambda \int_{\mathbf{F}} K_{s}\left(z, z^{\prime}\right) h\left(z^{\prime}, s\right) d \mu\left(z^{\prime}\right)
$$

for all $s \in \mathbf{S}^{\prime}$ then by Theorem 5.6

$$
h(z, s)=q(z, s)+\frac{\lambda}{D(\lambda)} \int_{\mathbf{F}} D_{\lambda}\left(z, z^{\prime}\right) q\left(z^{\prime}, s\right) d \mu\left(z^{\prime}\right)
$$

for all $s \in \mathbf{S}^{\prime}$, where $D(\lambda) \neq 0$. (We have assumed that $K_{s}\left(z, z^{\prime}\right)$ and $q(z, s)$ are uniformly bounded in S.) However, the right side of this last equation will be meromorphic in the larger domain $\mathbf{S}$, which is the method we shall use to obtain the analytic continuation of $E^{m, n}(z, s)$.

To apply Fredholm's Theorem to the integral equation (5.7) we need to carry out some steps to ensure that the kernel $K$ and function $q$ are bounded. The first is to take the difference of (5.7) at $a$ and $b$ (for $3<b<a)$ and eliminate the singularity at $z \equiv z^{\prime}$. Therefore, for $1<\operatorname{Re}(s)<b-2$,

$$
E_{\mathfrak{a}}^{m, n}(z, s) \nu_{a b}(s)=q^{m, n}(z, s)+\int_{\mathbf{F}} G_{a b}\left(z, z^{\prime}\right) E_{\mathfrak{a}}^{m, n}\left(z^{\prime}, s\right) d \mu\left(z^{\prime}\right)
$$

for

$$
q^{m, n}(z, s)=\sum_{\substack{0 \leq i \leq m \\
0 \leq i \leq m \\
(i, j) \neq(m, n)}}\left(\begin{array}{c}
m \\
i
\end{array}\right)\left(\begin{array}{c}
n \\
j
\end{array}\right) \int_{\mathbf{F}} G_{a b}^{m-i, n-j}\left(z, z^{\prime}\right) E_{\mathfrak{a}}^{i, j}\left(z^{\prime}, s\right) d \mu\left(z^{\prime}\right)
$$

when $\nu_{a b}(s)=(a(1-a)-s(1-s))^{-1}-(b(1-b)-s(1-s))^{-1}$.

The next step is to replace the kernel $G_{a b}\left(z, z^{\prime}\right)$ by a kernel $G_{a b}^{Y}\left(z, z^{\prime}\right)$ that has a growing term in $z^{\prime}$ removed for $z^{\prime}$ in cuspidal zones (these zones depend on a large parameter $Y$ ). This truncated kernel now has exponential decay as $z^{\prime}$ approaches cusps. When this new kernel is inserted into (5.9), terms involving $\phi_{\mathfrak{a} \mathfrak{b}}^{m, n}(s)$ appear which can be removed again by taking a certain linear combination at $Y, 2 Y$ and $4 Y$. The 
details of these computations are explained in depth in [11, Chapter 6] and [17, Section 3] so we omit the discussion. The end result of these computations is the identity

$$
h^{m, n}(z, s)=q_{2}^{m, n}(z, s)+\lambda(s) \int_{\mathbf{F}} H_{s}\left(z, z^{\prime}\right) h^{m, n}\left(z^{\prime}, s\right) d \mu\left(z^{\prime}\right)
$$

when $3<\operatorname{Re}(s)+2<b<a$ with

$$
\begin{aligned}
h^{m, n}(z, s) & =\left(2^{s-1+a}-1\right)\left(2^{s-1+b}-1\right) \nu_{a b}(s) E_{\mathfrak{a}}^{m, n}(z, s), \\
q_{2}^{m, n}(z, s) & =\left(2^{s-1+a}-1\right)\left(2^{s-1+b}-1\right) q^{m, n}(z, s), \\
\lambda(s) & =\frac{-1}{\nu_{a b}(s)}=\frac{(a-s)(a+s-1)(b-s)(b+s-1)}{(b-a)(a+b-1)} \\
H_{s}\left(z, z^{\prime}\right) & =\frac{\left(G_{a b}^{Y}-2^{s-1}\left(2^{a}+2^{b}\right) G_{a b}^{2 Y}+2^{2 s-2+a+b} G_{a b}^{4 Y}\right)\left(z, z^{\prime}\right)}{\left(2^{s-1+a}-1\right)\left(2^{s-1+b}-1\right)} .
\end{aligned}
$$

The kernel $H$ still has polynomial growth at cusps in the $z$ variable. The next step is to multiply through by $\eta(z)=e^{-\eta y_{\Gamma}(z)}$ with $0<\eta<2 \pi$ which controls the growth in $z$; furthermore, the restriction on $\eta$ ensures that the new kernel term $\left(\eta(z) \eta\left(z^{\prime}\right)^{-1} H_{s}\left(z, z^{\prime}\right)\right)$ appearing below in (5.13) is bounded. Therefore, for $(m, n) \neq(0,0)$, we obtain

$$
\eta(z) h^{m, n}(z, s)=\eta(z) q_{2}^{m, n}(z, s)+\lambda \int_{\mathbf{F}}\left(\eta(z) \eta\left(z^{\prime}\right)^{-1} H_{s}\left(z, z^{\prime}\right)\right)\left(\eta\left(z^{\prime}\right) h^{m, n}\left(z^{\prime}, s\right)\right) d \mu\left(z^{\prime}\right) .
$$

Again, a detailed argument regarding the computations behind (5.13) is given in [11] and [17]. Fredholm's Theorem will be applied to (5.13) in the course of Theorem 6.5 to achieve the meromorphic continuation of $h^{m, n}$ and hence $E^{m, n}$.

\section{The continuation}

\subsection{Proof of the meromorphic continuation of $E_{\mathfrak{a}}^{0,0}$}

In [11, Sections 6.1, 6.2] the meromorphic continuation of $E_{\mathfrak{a}}(z, s)$ is proved. The following proof is similar and tailored to extend to higher orders. We also supply some details omitted from the presentation in [11].

Let $\lambda$ be defined by (5.11) with fixed large constants $b<a$. Also define

$$
\mathbf{B}_{r}=\{s \in \mathbb{C}:|s| \leqslant r\} .
$$

Theorem 6.1. For every ball $\mathbf{B}_{r}$ of radius $r$ and nonzero $k \in \mathbf{Z}$, there exist functions $\phi_{\mathfrak{a} \mathfrak{b}}(s)$ and $\phi_{\mathfrak{a} \mathfrak{b}}(k, s)$ which are meromorphic on $\mathbf{B}_{r}$ as well as a function $A_{\mathfrak{a}}(s)$ which is analytic on $\mathbf{B}_{r}$ so that the following assertions hold for all $s \in \mathbf{B}_{r}:$

(i) We have the bounds

$$
\begin{aligned}
A_{\mathfrak{a}}(s) \phi_{\mathfrak{a} \mathfrak{b}}(s) & \ll 1, \\
A_{\mathfrak{a}}(s) \phi_{\mathfrak{a} \mathfrak{b}}(k, s) & \ll|k|^{\sigma}+|k|^{1-\sigma} .
\end{aligned}
$$

(ii) The Fourier expansion

$$
E_{\mathfrak{a}}\left(\sigma_{\mathfrak{b}} z, s\right)=\delta_{\mathfrak{a} \mathfrak{b}} y^{s}+\phi_{\mathfrak{a} \mathfrak{b}}(s) y^{1-s}+\sum_{k \neq 0} \phi_{\mathfrak{a} \mathfrak{b}}(k, s) W_{s}(k z)
$$

agrees with the definition (1.1) for $\operatorname{Re}(s)>1$, and, for all $z \in \mathbf{H}$, the series (6.1) converges to a meromorphic function for $s \in \mathbf{B}_{r}$.

(iii) We have the estimate

$$
A_{\mathfrak{a}}(s) E_{\mathfrak{a}}(z, s) \ll y_{\Gamma}(z)^{\sigma}+y_{\Gamma}(z)^{1-\sigma} .
$$

The implied constants in (i) and (iii) depend on $r$ and $\Gamma$ alone. 
Proof. Let

$$
h(z, s)=\frac{\left(2^{s-1+a}-1\right)\left(2^{s-1+b}-1\right)}{2^{2 s-1}-1} \nu_{a b}(s) E_{\mathfrak{a}}(z, s)
$$

and

$$
l(z, s)=\frac{2^{2 s-1+a-b}-1}{(2 b-1)(b-s)} Y^{s-b} E_{\mathfrak{a}}(z, b)-\frac{2^{2 s-1-a+b}-1}{(2 a-1)(a-s)} Y^{s-a} E_{\mathfrak{a}}(z, a) .
$$

Then it is shown in $[11,(6.8)]$, using the same method that proved (5.13), that

$$
\eta(z) h(z, s)=\eta(z) l(z, s)+\lambda \int_{\mathbf{F}}\left(\eta(z) \eta\left(z^{\prime}\right)^{-1} H_{s}\left(z, z^{\prime}\right)\right)\left(\eta\left(z^{\prime}\right) h\left(z^{\prime}, s\right)\right) d \mu\left(z^{\prime}\right)
$$

holds for $s \in \mathbf{B}_{r}$ when

$$
r=b-3 .
$$

(Note: (6.4) corrects a minor error in [11, p. 84].) By construction, $\eta(z) l(z, s)$ and $\eta(z) \eta\left(z^{\prime}\right)^{-1} H_{s}\left(z, z^{\prime}\right)$ are bounded for $(z, s) \in \mathbf{H} \times \mathbf{B}_{r}$ and $\left(z, z^{\prime}, s\right) \in \mathbf{H} \times \mathbf{F} \times \mathbf{B}_{r}$ respectively; therefore, it follows from Theorem 5.6 that

$$
\eta(z) h(z, s)=\eta(z) l(z, s)+\frac{\lambda}{D(\lambda)} \int_{\mathbf{F}} D_{\lambda}\left(z, z^{\prime}\right)\left(\eta\left(z^{\prime}\right) l\left(z^{\prime}, s\right)\right) d \mu\left(z^{\prime}\right)
$$

which is [11, (6.10)]. As shown in [11, Appendix A.4], $D(\lambda)$ is an analytic function of $s$ and $D_{\lambda}\left(z, z^{\prime}\right)$ is analytic in $s$, piecewise continuous in $z, z^{\prime}$ and bounded for $\left(z, z^{\prime}, s\right) \in \mathbf{H} \times \mathbf{F} \times \mathbf{B}_{r}$.

By combining (6.3) and (6.5) and setting

$$
\begin{aligned}
A_{\mathfrak{a}}(s) & =-\left(2^{s-1+a}-1\right)\left(2^{s-1+b}-1\right) D(\lambda), \\
A_{\mathfrak{a}}(z, s) & =\left(2^{2 s-1}-1\right) \lambda\left[D(\lambda) l(z, s)+\lambda \eta(z)^{-1} \int_{\mathbf{F}} \eta\left(z^{\prime}\right) D_{\lambda}\left(z, z^{\prime}\right) l\left(z^{\prime}, s\right) d \mu\left(z^{\prime}\right)\right]
\end{aligned}
$$

we have that $A_{\mathfrak{a}}(s)$ and $A_{\mathfrak{a}}(z, s)$ are analytic in $s$ and

$$
A_{\mathfrak{a}}(s) E_{\mathfrak{a}}(z, s)=A_{\mathfrak{a}}(z, s) .
$$

It also follows from (6.7) that

$$
A_{\mathfrak{a}}(z, s) \ll e^{\eta y_{\Gamma}(z)}
$$

for $s \in \mathbf{B}_{r}$ with an implicit constant depending only on $r$ and $\Gamma$.

We know the Fourier expansion (6.1) is valid for $\operatorname{Re}(s)>1$. So, for these $s$ values,

$$
\phi_{\mathfrak{a} \mathfrak{b}}(k, s)=\frac{1}{2 \sqrt{|k| y} K_{s-1 / 2}(2 \pi|k| y)} \int_{0}^{1} E_{\mathfrak{a}}\left(\sigma_{\mathfrak{b}}(x+i y), s\right) e^{-2 \pi i k x} d x .
$$

If we choose $y=1 / \sqrt{|k|}$ (for later convergence results) and replace $E_{\mathfrak{a}}(z, s)$ by $A_{\mathfrak{a}}(z, s) / A_{\mathfrak{a}}(s)$ in (6.9) we find

$$
\phi_{\mathfrak{a} \mathfrak{b}}(k, s)=\frac{1}{A_{\mathfrak{a}}(s) 2|k|^{1 / 4} K_{s-1 / 2}(2 \pi \sqrt{|k|})} \int_{0}^{1} A_{\mathfrak{a}}\left(\sigma_{\mathfrak{b}}(x+i / \sqrt{|k|}), s\right) e^{-2 \pi i k x} d x .
$$

This yields the meromorphic continuation of the Fourier coefficients $\phi_{\mathfrak{a} \mathfrak{b}}(k, s)$ (and similarly $\phi_{\mathfrak{a} \mathfrak{b}}(s)$ ) to all $s$ in $\mathbf{B}_{r}$. But we do not yet know that (6.1) holds in this larger domain. To show that it does, our task becomes proving bounds on $\phi_{\mathfrak{a} \mathfrak{b}}(k, s)$ that ensure (6.1) converges to a meromorphic function on $\mathbf{B}_{r}$.

In the arguments that follow we need additional estimates for the $K$-Bessel functions, which we state here. In Lemma 6.4 at the end of this section we will prove that

$$
2 \sqrt{u} K_{s-1 / 2}(2 \pi u) \ll e^{-2 \pi u}\left(u^{r+3}+u^{-r-3}\right)
$$

for all $u>0$ and $s$ in $\mathbf{B}_{r}$ with an implied constant that depends only on $r$. Also the asymptotic [11, (B.36)] shows that there exists an absolute constant $C$ so that, for any $s$ in $\mathbf{B}_{r}$ and $u \geqslant C r^{2}$,

$$
2 \sqrt{u} K_{s-1 / 2}(2 \pi u) \geqslant e^{-2 \pi u} / 2 .
$$


Continuing, we now apply Lemma 5.1 to equation (6.8) which produces the bound

$$
A_{\mathfrak{a}}\left(\sigma_{\mathfrak{b}}(x+i / \sqrt{|k|}), s\right) \ll e^{\eta\left(c_{\Gamma}+1 / c_{\Gamma}\right)\left(|k|^{1 / 2}+|k|^{-1 / 2}\right)} .
$$

Then using (6.12) and (6.13) in (6.10) we arrive at the inequality

$$
A_{\mathfrak{a}}(s) \phi_{\mathfrak{a} \mathfrak{b}}(k, s) \ll e^{2 \pi \sqrt{|k|}} \cdot e^{\eta\left(c_{\Gamma}+1 / c_{\Gamma}\right) \sqrt{|k|}}
$$

for $s \in \mathbf{B}_{r}$ and $|k| \geqslant C^{2} r^{4}$. all $k$,

Setting $y=C r^{2} /|k|$ in (6.9) instead of $y=|k|^{-1 / 2}$ and using (6.8), (6.12) and Lemma 5.1 shows that, for

$$
\begin{aligned}
A_{\mathfrak{a}}(s) \phi_{\mathfrak{a} \mathfrak{b}}(k, s) & \ll e^{2 \pi C r^{2}} \int_{0}^{1}\left|A_{\mathfrak{a}}\left(\sigma_{\mathfrak{b}}\left(x+i C r^{2} /|k|\right), s\right)\right| d x \\
& \ll e^{2 \pi C r^{2}} e^{\eta y_{\Gamma}\left(\sigma_{\mathfrak{b}}\left(x+i C r^{2} /|k|\right)\right.} \\
& \ll e^{2 \pi C r^{2}} e^{\eta\left(c_{\Gamma}+1 / c_{\Gamma}\right)\left[C r^{2} /|k|+|k| /\left(C r^{2}\right)\right]}
\end{aligned}
$$

for $s \in \mathbf{B}_{r}$ and implied constants depending on $r$ and $\Gamma$ alone. Therefore, for $|k|<C^{2} r^{4}$ we find that $A_{\mathfrak{a}}(s) \phi_{\mathfrak{a} \mathfrak{b}}(k, s) \ll 1$. Hence, for all $k \neq 0$,

$$
A_{\mathfrak{a}}(s) \phi_{\mathfrak{a} \mathfrak{b}}(k, s) \ll e^{2 \pi D \sqrt{|k|}}
$$

with $D=1+c_{\Gamma}+1 / c_{\Gamma}$. Similar arguments show that

$$
A_{\mathfrak{a}}(s) \phi_{\mathfrak{a} \mathfrak{b}}(s) \ll 1
$$

Next employ the estimate (6.11), which is proved in Lemma 6.4, to see that

$$
\left|W_{s}(k z)\right| \ll e^{-2 \pi|k| y}\left((|k| y)^{r+3}+(|k| y)^{-r-3}\right)
$$

for $s \in \mathbf{B}_{r}$. As a consequence of this inequality and (6.15)

$$
\begin{aligned}
A_{\mathfrak{a}}(s) \sum_{k \neq 0} \phi_{\mathfrak{a} \mathfrak{b}}(k, s) W_{s}(k z) & \ll \sum_{k=1}^{\infty}\left((k y)^{r+3}+(k y)^{-r-3}\right) e^{2 \pi[D \sqrt{k}-k y]} \\
& \ll e^{-2 \pi y}\left[y^{r+3}+\frac{1}{y^{r+3}}+e^{8 \pi D^{2} / y}\left(\frac{1}{y^{r-1}}+\frac{1}{y^{r+5}}\right)+e^{-\pi y}\left(y^{2}+\frac{1}{y^{r+4}}\right)\right] .
\end{aligned}
$$

The last estimate (6.17) follows from Lemma 6.2 at the end of this section. We now see that, for all $z \in \mathbf{H}$, (6.1) converges uniformly to a meromorphic function of $s \in \mathbf{B}_{r}$, proving part (ii) of the theorem. Also (6.17) implies that

$$
A_{\mathfrak{a}}\left(\sigma_{\mathfrak{b}} z, s\right)=A_{\mathfrak{a}}(s) E_{\mathfrak{a}}\left(\sigma_{\mathfrak{b}} z, s\right)=A_{\mathfrak{a}}(s)\left(\delta_{\mathfrak{a} \mathfrak{b}} y^{s}+\phi_{\mathfrak{a} \mathfrak{b}}(s) y^{1-s}\right)+O\left(e^{-2 \pi y} y^{r+3}\right)
$$

as $y \rightarrow \infty$ and, consequently,

$$
A_{\mathfrak{a}}(z, s)=A_{\mathfrak{a}}(s) E_{\mathfrak{a}}(z, s) \ll y_{\Gamma}(z)^{|\sigma-1 / 2|+1 / 2}
$$

for all $z \in \mathbf{H}$. The bound (6.19) is a significant improvement of (6.8), and part (iii) of the theorem follows easily from (6.19). Using (6.19) in (6.14) with Lemma 5.1 produces

$$
\begin{aligned}
A_{\mathfrak{a}}(s) \phi_{\mathfrak{a} \mathfrak{b}}(k, s) & \ll|k|^{|\sigma-1 / 2|+1 / 2}+|k|^{-|\sigma-1 / 2|-1 / 2} \\
& \ll|k|^{\sigma}+|k|^{1-\sigma} .
\end{aligned}
$$

This bound, along with (6.16), shows part $(i)$ of the theorem and the proof of Theorem 6.1 is complete. 
Lemma 6.2. For any $D, r \geqslant 0$ and $y>0$

$$
\sum_{k=1}^{\infty} k^{r} e^{2 \pi(D \sqrt{k}-y k)} \ll e^{-2 \pi y}\left(1+\frac{e^{-\pi y}}{y^{r+1}}+\frac{e^{8 \pi D^{2} / y}}{y^{2 r+2}}\right)
$$

for an implied constant depending on $r$ and $D$ alone.

Proof. We may write the left side above as

$$
e^{2 \pi(D-y)}+\sum_{k=1}^{\infty}(k+1)^{r} e^{2 \pi(D \sqrt{k+1}-y k)} e^{-2 \pi y}
$$

which is bounded by

$$
e^{-2 \pi y}\left(e^{2 \pi D}+\sum_{k=1}^{\infty}(2 k)^{r} e^{2 \pi(D \sqrt{2 k}-y k)}\right) .
$$

The upper bound $\sqrt{2} D \sqrt{k}-y k \leqslant-y k / 2$ holds if and only if $k \geqslant 8 D^{2} / y^{2}$ so

$$
\begin{aligned}
\sum_{k=1}^{\infty} k^{r} e^{2 \pi(\sqrt{2} D \sqrt{k}-y k)} & =\sum_{k \leqslant 8 D^{2} / y^{2}} k^{r} e^{2 \pi(\sqrt{2} D \sqrt{k}-y k)}+\sum_{k>8 D^{2} / y^{2}} k^{r} e^{2 \pi(\sqrt{2} D \sqrt{k}-y k)} \\
& \leqslant \sum_{k \leqslant 8 D^{2} / y^{2}}\left(8 D^{2} / y^{2}\right)^{r} e^{8 \pi D^{2} / y}+\sum_{k>8 D^{2} / y^{2}} k^{r} e^{-\pi k y} \\
& \leqslant\left(\frac{8 D^{2}}{y^{2}}\right)^{r+1} e^{8 \pi D^{2} / y}+\sum_{k=1}^{\infty} k^{r} e^{-\pi k y}
\end{aligned}
$$

Use the formula

$$
\int_{0}^{\infty} e^{-y t} t^{s-1} d t=\frac{\Gamma(s)}{y^{s}}
$$

for $\operatorname{Re}(s)>0$, as in $[2,(2.4)]$, to see that

$$
\sum_{k=1}^{\infty} k^{r} e^{-\pi k y} \leqslant e^{-\pi y}\left(1+2^{r} e^{-\pi y}+\frac{3^{r} \Gamma(r+1)}{(\pi y)^{r+1}}\right)
$$

for all $r \geqslant 0$. The proof is completed by combining inequalities (6.20), (6.21) and (6.22).

Lemma 6.3. For $\sigma=\operatorname{Re}(s) \geqslant 1$ and $y>0$,

$$
\left|K_{s-1 / 2}(y)\right| \leqslant \kappa(s) e^{-y}\left(y^{\sigma-1 / 2}+y^{-\sigma+1 / 2}\right)
$$

where $\kappa(s)$ is the continuous function $\kappa(s)=\pi^{1 / 2} 3^{\sigma-1}(1+|\Gamma(2 \sigma-1)|) /|\Gamma(s)|$.

Proof. For $\sigma>0$ and $y>0$ we have the integral representation

$$
K_{s-1 / 2}(y)=\frac{\sqrt{\pi}}{\Gamma(s)}\left(\frac{y}{2}\right)^{s-1 / 2} \int_{1}^{\infty}\left(t^{2}-1\right)^{s-1} e^{-t y} d t
$$

from [11, p. 205]. Suppose $\sigma \geqslant 1$. It is easy to see that

$$
\left|\int_{1}^{2}(t-1)^{s-1}(t+1)^{s-1} e^{-t y} d t\right| \leqslant 3^{\sigma-1} e^{-y}
$$

and

$$
\begin{aligned}
\left|\int_{2}^{\infty}(t-1)^{s-1}(t+1)^{s-1} e^{-t y} d t\right| & \leqslant e^{-y} \int_{1}^{\infty} u^{\sigma-1}(u+2)^{\sigma-1} e^{-u y} d u \\
& \leqslant e^{-y} 3^{\sigma-1} \int_{0}^{\infty} u^{2 \sigma-2} e^{-u y} d u \\
& \leqslant e^{-y} 3^{\sigma-1} \Gamma(2 \sigma-1) y^{1-2 \sigma}
\end{aligned}
$$

Putting these estimates together finishes the proof. 
Lemma 6.4. For all $r, y>0$ and all $s \in \mathbf{B}_{r}$

$$
W_{s}(y):=2 \sqrt{y} K_{s-1 / 2}(2 \pi y) \ll e^{-2 \pi y}\left(y^{r+3}+y^{-r-3}\right)
$$

with an implied constant that depends solely on $r$.

Proof. Denote by $\mathcal{S}_{m}$ the vertical strip of all $s \in \mathbf{C}$ with $m \leqslant \operatorname{Re}(s)<m+1$. It follows from Lemma 6.3 that

$$
\begin{aligned}
& \left|K_{s-1 / 2}(y)\right| \leqslant \kappa_{2}(s) e^{-y}\left(y^{5 / 2}+y^{-5 / 2}\right) \text { when } s \in \mathcal{S}_{2}, \\
& \left|K_{s-1 / 2}(y)\right| \leqslant \kappa_{1}(s) e^{-y}\left(y^{3 / 2}+y^{-3 / 2}\right) \text { when } s \in \mathcal{S}_{1} .
\end{aligned}
$$

for continuous functions $\kappa_{i}(s)$. Then, using the identity [11, p. 204],

$$
K_{s-1 / 2}(y)=\frac{2 s+1}{y} K_{s+1 / 2}(y)-K_{s+3 / 2}(y)
$$

we find

$$
\left|K_{s-1 / 2}(y)\right| \leqslant \kappa_{0}(s) e^{-y}\left(y^{5 / 2}+y^{-5 / 2}\right) \quad \text { when } s \in \mathcal{S}_{0}
$$

and continuing we get

$$
\left|K_{s-1 / 2}(y)\right| \leqslant \kappa_{-1}(s) e^{-y}\left(y^{3 / 2}+y^{-7 / 2}\right) \quad \text { when } s \in \mathcal{S}_{-1}
$$

and in general, for positive integers $m$,

$$
\left|K_{s-1 / 2}(y)\right| \leqslant \kappa_{-m}(s) e^{-y}\left(y^{5 / 2}+y^{-m-5 / 2}\right) \quad \text { when } s \in \mathcal{S}_{-m} .
$$

Therefore, for all $r>0$ and $s \in \mathbf{B}_{r}$,

$$
\left|K_{s-1 / 2}(y)\right| \ll e^{-y}\left(y^{r+5 / 2}+y^{-r-7 / 2}\right)
$$

and the proof follows.

\subsection{Proof of the meromorphic continuation of $E^{m, n}$}

One of the main results in this paper is the generalization of Theorem 6.1 to higher orders.

Theorem 6.5. For every ball $\mathbf{B}_{r}$ of radius $r$, nonzero integer $k \in \mathbf{Z}$, and all integers $m, n \geqslant 0$ there exist functions $\phi_{\mathfrak{a} \mathfrak{b}}^{m, n}(s)$ and $\phi_{\mathfrak{a} \mathfrak{b}}^{m, n}(k, s)$ which are meromorphic on $\mathbf{B}_{r}$, as well as a function $A_{\mathfrak{a}}(s)$ which is analytic on $\mathbf{B}_{r}$ so that the following assertions hold for all $s \in \mathbf{B}_{r}$ :

(i) We have the bounds

$$
\begin{aligned}
A_{\mathfrak{a}}^{m+n+1}(s) \phi_{\mathfrak{a} \mathfrak{b}}^{m, n}(s) & \ll 1 \\
A_{\mathfrak{a}}^{m+n+1}(s) \phi_{\mathfrak{a} \mathfrak{b}}^{m, n}(k, s) & \ll\left(\log ^{m+n}|k|+1\right)\left(|k|^{\sigma}+|k|^{1-\sigma}\right) .
\end{aligned}
$$

(ii) The Fourier expansion

$$
E_{\mathfrak{a}}^{m, n}\left(\sigma_{\mathfrak{b}} z, s\right)=\delta_{0,0}^{m, n} \cdot \delta_{\mathfrak{a} \mathfrak{b}} y^{s}+\phi_{\mathfrak{a} \mathfrak{b}}^{m, n}(s) y^{1-s}+\sum_{k \neq 0} \phi_{\mathfrak{a} \mathfrak{b}}^{m, n}(k, s) W_{s}(k z)
$$

agrees with the definition (1.1) for $\operatorname{Re}(s)>1$ and, for all $z \in \mathbf{H}$, converges to a meromorphic function for $s \in \mathbf{B}_{r}$.

(iii) We have the estimate

$$
A_{\mathfrak{a}}^{m+n+1}(s) E_{\mathfrak{a}}^{m, n}(z, s) \ll y_{\mathbf{F}}(z)^{|\sigma-1 / 2|+1 / 2} .
$$

The implied constants in (i) and (iii) depend on $r, m, n, f, g$ and $\Gamma$ alone. 
Proof. Our proof involves induction on $m+n$ with the first step given in Theorem 6.1. The remainder of this proof establishes the induction step.

To begin, we shall now make an additional adjustment to (5.13) in order to eliminate possible poles in $s$. Set $q_{A}^{m, n}(z, s)=A_{\mathfrak{a}}(s)^{m+n} q_{2}^{m, n}(z, s)$ and $h_{A}^{m, n}(z, s)=A_{\mathfrak{a}}(s)^{m+n} h^{m, n}(z, s)$. For $1<\operatorname{Re}(s)$ and $s \in \mathbf{B}_{r}$ (with $r=b-3$ as before),

$$
\eta(z) h_{A}^{m, n}(z, s)=\eta(z) q_{A}^{m, n}(z, s)+\lambda \int_{\mathbf{F}}\left(\eta(z) \eta\left(z^{\prime}\right)^{-1} H_{s}\left(z, z^{\prime}\right)\right)\left(\eta\left(z^{\prime}\right) h_{A}^{m, n}\left(z^{\prime}, s\right)\right) d \mu\left(z^{\prime}\right) .
$$

We need to check that $\eta(z) q_{A}^{m, n}(z, s)$ is bounded before we may apply Fredholm's Theorem (Theorem 5.6) to (6.25). Using Proposition 5.5 and, by induction, part (iii) of the theorem,

$$
\begin{aligned}
q_{A}^{m, n}(z, s) & =A_{\mathfrak{a}}(s)^{m+n}\left(2^{s-1+a}-1\right)\left(2^{s-1+b}-1\right) \sum_{\substack{0 \leq i \leq m \\
0 \leq i \leq m \\
(i, j) \neq(m, n)}}\left(\begin{array}{c}
m \\
i
\end{array}\right)\left(\begin{array}{c}
n \\
j
\end{array}\right) \int_{\mathbf{F}} G_{a b}^{m-i, n-j}\left(z, z^{\prime}\right) E_{\mathfrak{a}}^{i, j}\left(z^{\prime}, s\right) d \mu\left(z^{\prime}\right) \\
& \ll \sum_{\substack{0 \leq i \leq m \\
0 \leq i \leq m \\
i, j) \neq(m, n)}}\left|A_{\mathfrak{a}}(s)^{m+n-i-j-1}\right| \int_{\mathbf{F}}\left|G_{a b}^{m-i, n-j}\left(z, z^{\prime}\right) A_{\mathfrak{a}}(s)^{i+j+1} E_{\mathfrak{a}}^{i, j}\left(z^{\prime}, s\right)\right| d \mu\left(z^{\prime}\right) \\
& \ll \sum_{\substack{0 \leq i \leq m \\
0 \leq i \leq m \\
(i, j) \neq(m, n)}} \int_{\mathbf{F}}\left|y_{\mathbf{F}}(z)^{1-b+\epsilon} y_{\mathbf{F}}\left(z^{\prime}\right)^{1-b+\epsilon}\left(y_{\mathbf{F}}(z)^{\sigma}+y_{\mathbf{F}}(z)^{1-\sigma}\right)\right| d \mu\left(z^{\prime}\right) \\
& \ll 1 .
\end{aligned}
$$

In particular, the function $q_{A}^{m, n}(z, s)$ is bounded for $(z, s) \in \mathbf{F} \times \mathbf{B}_{r}$ and continuous in the $z$ variable. We can now apply Fredholm's theorem to (6.25) with the result

$$
h_{A}^{m, n}(z, s)=q_{A}^{m, n}(z, s)+\frac{\lambda}{D(\lambda)} \int_{\mathbf{F}} \eta(z)^{-1} \eta\left(z^{\prime}\right) D_{\lambda}\left(z, z^{\prime}\right) q_{A}^{m, n}\left(z^{\prime}, s\right) d \mu\left(z^{\prime}\right)
$$

for each $s \in \mathbf{B}_{r}$ such that $D(\lambda) \neq 0$. Hence, if we set

$$
A_{\mathfrak{a}}^{m, n}(z, s)=-\lambda\left(D(\lambda) q_{A}^{m, n}(z, s)+\lambda \eta(z)^{-1} \int_{\mathbf{F}} \eta\left(z^{\prime}\right) D_{\lambda}\left(z, z^{\prime}\right) q_{A}^{m, n}\left(z^{\prime}, s\right) d \mu\left(z^{\prime}\right)\right)
$$

we find $A_{\mathfrak{a}}^{m+n+1}(s) E_{\mathfrak{a}}^{m, n}(z, s)=A_{\mathfrak{a}}^{m, n}(z, s)$. Also the $\eta(z)^{-1}$ term in (6.27) implies that

$$
A_{\mathfrak{a}}^{m+n+1}(s) E_{\mathfrak{a}}^{m, n}(z, s) \ll e^{\eta y_{\Gamma}(z)} .
$$

Now we argue as in Theorem 6.1, the only complication being that $E_{\mathfrak{a}}^{m, n}$ is not automorphic. Since (6.28) is also true for all smaller $m, n$ by induction, we have that

$$
A_{\mathfrak{a}}^{m+n+1}(s) Q_{\mathfrak{a}}^{m, n}(z, s) \ll \log ^{m+n}\left(y_{\mathbf{F}}(z)+e\right) \cdot e^{\eta y_{\Gamma}(z)}
$$

for $z \in \mathbf{F}$. Hence, for all $z \in \mathbf{H}$,

$$
A_{\mathfrak{a}}^{m+n+1}(s) Q_{\mathfrak{a}}^{m, n}(z, s) \ll \log ^{m+n}\left(y_{\Gamma}(z)+e\right) \cdot e^{\eta y_{\Gamma}(z)}
$$

since the left side is automorphic and $\log ^{m+n}\left(y_{\mathbf{F}}(z)+e\right)$ is an increasing function of $y_{\mathbf{F}}(z)$. With Lemma 5.1,

$$
\begin{aligned}
A_{\mathfrak{a}}^{m+n+1}(s) Q_{\mathfrak{a}}^{m, n}\left(\sigma_{\mathfrak{b}} z, s\right) & \ll \log ^{m+n}\left(\left(c_{\Gamma}+1 / c_{\Gamma}\right)(y+1 / y)+e\right) \cdot e^{\eta\left(c_{\Gamma}+1 / c_{\Gamma}\right)(y+1 / y)} \\
& \ll \log ^{m+n}(y+1 / y) \cdot e^{2 \pi\left(c_{\Gamma}+1 / c_{\Gamma}\right)(y+1 / y)} .
\end{aligned}
$$

Consequently, with (5.3) and for all $z \in \mathbf{H}$,

$$
\begin{aligned}
A_{\mathfrak{a}}^{m+n+1}(s) E_{\mathfrak{a}}^{m, n}\left(\sigma_{\mathfrak{b}} z, s\right) & \ll \log ^{m+n}(y+1 / y) \cdot e^{2 \pi\left(c_{\Gamma}+1 / c_{\Gamma}\right)(y+1 / y)} \\
& \ll e^{2 \pi D^{\prime}(y+1 / y)}
\end{aligned}
$$


for $D^{\prime}=c_{\Gamma}+1 / c_{\Gamma}+1$, say. Now arguing similarly to (6.9) - (6.16) we see that the Fourier coefficients of $E_{\mathfrak{a}}^{m, n}$ may be continued and satisfy the bounds

$$
\begin{aligned}
A_{\mathfrak{a}}^{m+n+1}(s) \phi_{\mathfrak{a} \mathfrak{b}}^{m, n}(s) & \ll 1 \\
A_{\mathfrak{a}}^{m+n+1}(s) \phi_{\mathfrak{a} \mathfrak{b}}^{m, n}(k, s) & \ll e^{2 \pi D^{\prime \prime} \sqrt{|k|}}
\end{aligned}
$$

with $D^{\prime \prime}=2+c_{\Gamma}+1 / c_{\Gamma}$. Thus, as in (6.17), we have shown that (6.23) converges to a meromorphic function for $s \in \mathbf{B}_{r}$ and that

$$
A_{\mathfrak{a}}^{m+n+1}(s) E_{\mathfrak{a}}^{m, n}(z, s) \ll y_{\mathbf{F}}(z)^{|\sigma-1 / 2|+1 / 2} .
$$

Inductively, it has been shown that the bound (6.29) also is true for all smaller $m, n$ and therefore, again with (5.2),

$$
A_{\mathfrak{a}}^{m+n+1}(s) Q_{\mathfrak{a}}^{m, n}(z, s) \ll \log ^{m+n}\left(y_{\mathbf{F}}(z)+e\right) \cdot y_{\mathbf{F}}(z)^{|\sigma-1 / 2|+1 / 2} .
$$

The above result is true with $y_{\mathbf{F}}$ replaced by $y_{\Gamma}$ and, employing Lemma 5.1 once more,

$$
A_{\mathfrak{a}}^{m+n+1}(s) Q_{\mathfrak{a}}^{m, n}\left(\sigma_{\mathfrak{b}} z, s\right) \ll \log ^{m+n}(y+1 / y) \cdot(y+1 / y)^{|\sigma-1 / 2|+1 / 2} .
$$

With (5.3) it is now true that

$$
A_{\mathfrak{a}}^{m+n+1}(s) E_{\mathfrak{a}}^{m, n}\left(\sigma_{\mathfrak{b}} z, s\right) \ll \log ^{m+n}(y+1 / y) \cdot(y+1 / y)^{|\sigma-1 / 2|+1 / 2} .
$$

Use this in the analog of (6.14) to finally see that

$$
A_{\mathfrak{a}}^{m+n+1}(s) \phi_{\mathfrak{a} \mathfrak{b}}^{m, n}(k, s) \ll\left(\log ^{m+n}|k|+1\right)\left(|k|^{\sigma}+|k|^{1-\sigma}\right) .
$$

All parts of the theorem have been completed, establishing the induction.

We have a straightforward corollary to the above results.

Corollary 6.6. For every ball $\mathbf{B}_{r}$ of radius $r$ and all integers $m, n \geqslant 0$ the following hold for all $s \in \mathbf{B}_{r}, z \in \mathbf{H}$ and $\tau \in \Gamma$ :

$$
\begin{aligned}
\Delta E_{\mathfrak{a}}^{m, n}(z, s) & =s(1-s) E_{\mathfrak{a}}^{m, n}(z, s), \\
E_{\mathfrak{a}}^{m, n}(\tau z, s) & =\sum_{i=0}^{m} \sum_{j=0}^{n}\left(\begin{array}{c}
m \\
i
\end{array}\right)\left(\begin{array}{c}
n \\
j
\end{array}\right) S_{m-i, n-j}(\tau) E_{\mathfrak{a}}^{i, j}(z, s) \\
E_{\mathfrak{a}}^{m, n}\left(\sigma_{\mathfrak{b}} z, s\right) & =\delta_{0,0}^{m, n} \cdot \delta_{\mathfrak{a} \mathfrak{b}} y^{s}+\phi_{\mathfrak{a} \mathfrak{b}}^{m, n}(s) y^{1-s}+O\left(e^{-2 \pi y}\right) \text { as } y \rightarrow \infty .
\end{aligned}
$$

The implied constant in (iii) will depend on $r, m, n, f, g$ and $\Gamma$ alone.

Proof. To prove (6.30) apply the Laplacian $\Delta$ directly to the Fourier expansion (6.32). The relation (6.31) clearly follows from (4.2) by analytic continuation. To show (6.32) use part (i) of Theorem 6.5,

$$
W_{s}(k z) \ll e^{-2 \pi k y}
$$

for $k y \geqslant r^{2}$ (which follows from the asymptotic [11, (B.36)]) and Lemma 6.4.

\section{The functional equation}

We now prove that $\mathcal{E}^{m, n}$ satisfies the functional equations stated in (2.7) and (2.8) which will yield the proof of Theorem 2.2. Recall from Theorem 6.5 that $E_{\mathfrak{a}}^{m, n}(z, s)$ and $\phi_{\mathfrak{a} \mathfrak{b}}^{m, n}(s)$ are meromorphic functions of $s \in \mathbf{C}$.

Theorem 7.1. For all $m, n \geqslant 0$ and all $s \in \mathbf{C}, z \in \mathbf{H}$

$$
\begin{aligned}
\sum_{\substack{0 \leqslant i \leqslant m \\
0 \leqslant j \leqslant n}}\left(\begin{array}{c}
m \\
i
\end{array}\right)\left(\begin{array}{c}
n \\
j
\end{array}\right) \Phi^{i, j}(1-s) \mathcal{E}^{m-i, n-j}(z, s) & =\mathcal{E}^{m, n}(z, 1-s) \\
\sum_{\substack{0 \leqslant i \leqslant m \\
0 \leqslant j \leqslant n}}\left(\begin{array}{c}
m \\
i
\end{array}\right)\left(\begin{array}{c}
n \\
j
\end{array}\right) \Phi^{i, j}(1-s) \Phi^{m-i, n-j}(s) & = \begin{cases}\mathrm{I}_{r} & \text { if } m=n=0, \\
0 & \text { otherwise. }\end{cases}
\end{aligned}
$$


Proof. First set

$$
\mathcal{J}^{m, n}(z, s):=\sum_{\substack{0 \leqslant i \leqslant m \\
0 \leqslant j \leqslant n}}\left(\begin{array}{c}
m \\
i
\end{array}\right)\left(\begin{array}{c}
n \\
j
\end{array}\right) \Phi^{i, j}(1-s) \mathcal{E}^{m-i, n-j}(z, s) .
$$

When $\gamma \in \Gamma$ we see by (6.31) that

$$
\begin{aligned}
\mathcal{J}^{m, n}(\gamma z, s) & =\sum_{\substack{0 \leqslant i \leqslant m \\
0 \leqslant j \leqslant n}}\left(\begin{array}{c}
m \\
i
\end{array}\right)\left(\begin{array}{l}
n \\
j
\end{array}\right) \Phi^{i, j}(1-s) \sum_{\substack{0 \leqslant c \leqslant m-i \\
0 \leqslant d \leqslant n-j}}\left(\begin{array}{c}
m-i \\
c
\end{array}\right)\left(\begin{array}{c}
n-j \\
d
\end{array}\right) S_{m-i-c, n-j-d}(\gamma) \mathcal{E}^{c, d}(z, s) \\
& =\sum_{\substack{0 \leqslant c \leqslant m \\
0 \leqslant d \leqslant n}}\left(\begin{array}{c}
m \\
c
\end{array}\right)\left(\begin{array}{c}
n \\
d
\end{array}\right) S_{m-c, n-d}(\gamma) \sum_{\substack{0 \leqslant i \leqslant c \\
0 \leqslant j \leqslant d}}\left(\begin{array}{c}
c \\
i
\end{array}\right)\left(\begin{array}{c}
d \\
j
\end{array}\right) \Phi^{i, j}(1-s) \mathcal{E}^{c-i, d-j}(z, s) \\
& =\sum_{\substack{0 \leqslant s \leqslant m \\
0 \leqslant d \leqslant n}}\left(\begin{array}{c}
m \\
c
\end{array}\right)\left(\begin{array}{l}
n \\
d
\end{array}\right) S_{m-c, n-d}(\gamma) \mathcal{J}^{c, d}(z, s) .
\end{aligned}
$$

To prove the theorem we use induction on $m+n$ with the base case, $(m, n)=(0,0)$, being the well-known functional equation for the classical non-holomorphic Eisenstein series. For the induction step we now assume that

$$
\mathcal{E}^{c, d}(z, 1-s)=\mathcal{J}^{c, d}(z, s)
$$

for all $c, d$ with $c+d<N$ and suppose that $m+n=N$. Combine (7.3) and (7.4) to see that

$$
\begin{aligned}
\mathcal{J}^{m, n}(\gamma z, s) & =\mathcal{J}^{m, n}(z, s)+\sum_{\substack{0 \leq c \leq m \\
0 \leq d \leq n \\
(c, d) \neq(m, n)}}\left(\begin{array}{c}
m \\
c
\end{array}\right)\left(\begin{array}{l}
n \\
d
\end{array}\right) S_{m-c, n-d}(\gamma) \mathcal{J}^{c, d}(z, s) \\
& =\mathcal{J}^{m, n}(z, s)+\mathcal{E}^{m, n}(\gamma z, 1-s)-\mathcal{E}^{m, n}(z, 1-s) .
\end{aligned}
$$

Therefore the function $\mathcal{J}^{m, n}(z, s)-\mathcal{E}^{m, n}(z, 1-s)$ is $\Gamma$-invariant in the $z$ variable. We also see from (6.30) that $\mathcal{J}^{m, n}(z, s)$ is an eigenfunction on the Laplacian with eigenvalue $s(1-s)$ and, with (6.24), must be bounded by $y_{\Gamma}(z)^{|\sigma-1 / 2|+1 / 2}$. Therefore, by a result of Selberg (see [11, Lemma 6.4]), $\mathcal{J}^{m, n}(z, s)-\mathcal{E}^{m, n}(z, 1-s)$ is equal to a linear combination of the classical $(m=n=0)$ non-holomorphic Eisenstein series:

$$
\mathcal{J}^{m, n}(z, s)-\mathcal{E}^{m, n}(z, 1-s)=\Psi^{m, n}(s) \mathcal{E}^{0,0}(z, s)
$$

for some $r \times r$ matrix $\Psi^{m, n}(s)$. Considering the Fourier expansions of both sides of (7.5) and comparing the coefficients of $y^{s}$ shows that

$$
\Psi^{m, n}(s)=0
$$

and proves (7.1). Comparing the coefficients of $y^{1-s}$ then yields (7.2). We have now completed the induction step and the proof is complete.

\section{Further examples}

Consider a group $\Gamma$ with $r$ cusps. Taking $(m, n)=(2,1)$, the action by $\Gamma$ as described in Theorem 2.1 becomes the identity

$$
\left(\begin{array}{l}
\mathcal{E}^{2,1} \\
\mathcal{E}^{2,0} \\
\mathcal{E}^{1,1} \\
\mathcal{E}^{1,0} \\
\mathcal{E}^{0,1} \\
\mathcal{E}^{0,0}
\end{array}\right)(\gamma z, s)=\bar{\chi}(\gamma)\left(\begin{array}{cccccc}
1 & -\overline{\langle\gamma, g\rangle} & -2\langle\gamma, f\rangle & 2\langle\gamma, f\rangle \overline{\langle\gamma, g\rangle} & \langle\gamma, f\rangle^{2} & -\langle\gamma, f\rangle^{2} \overline{\langle\gamma, g\rangle} \\
& 1 & & -2\langle\gamma, f\rangle & & \langle\gamma, f\rangle^{2} \\
& & 1 & -\overline{\langle\gamma, g\rangle} & -\langle\gamma, f\rangle & \langle\gamma, f\rangle \overline{\langle\gamma, g\rangle} \\
& & & 1 & & -\langle\gamma, f\rangle \\
& & & & 1 & -\overline{\langle\gamma, g\rangle}
\end{array}\right)\left(\begin{array}{l}
\mathcal{E}^{2,1} \\
\mathcal{E}^{2,0} \\
\mathcal{E}^{1,1} \\
\mathcal{E}^{1,0} \\
\mathcal{E}^{0,1} \\
\mathcal{E}^{0,0}
\end{array}\right)(z, s)
$$


where, as in (4.6), each matrix entry above is multiplied by the $r \times r$ identity matrix $\mathrm{I}_{r}$. The functional equation in Theorem 2.2 is the relation

$$
\left(\begin{array}{l}
\mathcal{E}^{2,1} \\
\mathcal{E}^{2,0} \\
\mathcal{E}^{1,1} \\
\mathcal{E}^{1,0} \\
\mathcal{E}^{0,1} \\
\mathcal{E}^{0,0}
\end{array}\right)(z, 1-s)=\left(\begin{array}{cccccc}
\Phi^{0,0} & \Phi^{0,1} & 2 \Phi^{1,0} & 2 \Phi^{1,1} & \Phi^{2,0} & \Phi^{2,1} \\
& \Phi^{0,0} & & 2 \Phi^{1,0} & & \Phi^{2,0} \\
& & \Phi^{0,0} & \Phi^{0,1} & \Phi^{1,0} & \Phi^{1,1} \\
& & & \Phi^{0,0} & & \Phi^{1,0} \\
& & & & \Phi^{0,0} & \Phi^{0,1} \\
& & & & & \Phi^{0,0}
\end{array}\right)(1-s)\left(\begin{array}{l}
\mathcal{E}^{2,1} \\
\mathcal{E}^{2,0} \\
\mathcal{E}^{1,1} \\
\mathcal{E}^{1,0} \\
\mathcal{E}^{0,1} \\
\mathcal{E}^{0,0}
\end{array}\right)(z, s)
$$

As throughout the paper, each entry $\mathcal{E}$ is a column vector of size $r \times 1$, and each entry $\Phi$ is an $r \times r$ matrix.

Consider the specific example when $\Gamma=\Gamma_{0}(p)$ is the Hecke congruence group of prime level $p$. The group $\Gamma_{0}(p)$ has two inequivalent cusps which my be uniformized to be at $\infty$ and 0 . In this case, we have that for each $(m, n)$, the vector $\mathcal{E}^{m, n}$ is simply the order pair ${ }^{t}\left(E_{\infty}^{m, n}, E_{0}^{m, n}\right)$. Taking $(m, n)=(2,0)$, and omitting $z$ from the notation, Theorem 2.2 yields the identity

$$
\left(\begin{array}{l}
E_{\infty}^{2,0} \\
E_{0}^{2,0} \\
E_{\infty}^{1,0} \\
E_{0}^{1,0} \\
E_{\infty}^{0,0} \\
E_{0}^{0,0}
\end{array}\right)(1-s)=\left(\begin{array}{cccccc}
\Phi_{\infty}^{0,0} & \Phi_{\infty}^{0,0} & 2 \Phi_{\infty}^{1,0} & 2 \Phi_{\infty 0}^{1,0} & \Phi_{\infty}^{2,0} & \Phi_{\infty 00}^{2,0} \\
\Phi_{0 \infty}^{0,0} & \Phi_{00}^{0,0} & 2 \Phi_{0 \infty}^{1,0} & 2 \Phi_{00}^{1,0} & \Phi_{0 \infty}^{2,0} & \Phi_{00}^{2,0} \\
& & \Phi_{\infty}^{0,0} & \Phi_{\infty}^{0,0} & \Phi_{\infty}^{1,0} & \Phi_{\infty}^{1,0} \\
& & \Phi_{0 \infty}^{0,0} & \Phi_{00}^{0,0} & \Phi_{0 \infty}^{1,0} & \Phi_{00}^{1,0} \\
& & & & \Phi_{\infty}^{0,0} & \Phi_{\infty}^{0,0} \\
& & & & \Phi_{0 \infty}^{0,0} & \Phi_{00}^{0,0}
\end{array}\right)(1-s)\left(\begin{array}{l}
E_{\infty}^{2,0} \\
E_{0}^{2,0} \\
E_{\infty}^{1,0} \\
E_{0}^{1,0} \\
E_{\infty}^{0,0} \\
E_{0}^{0,0}
\end{array}\right)(s)
$$

As in [17], each entry in the scattering matrix in (8.1) can be expressed as a type of Kloosterman sum twisted by modular symbols. It certainly would be interesting to find explicit formulas for the entries of (8.1). The matrix identity (2.6) implies a number of relations for these scattering matrix entries.

\section{A Invariant height}

For any Fuchsian group $\Gamma$ we define a constant $c_{\Gamma}>0$ as follows. Let

$$
\mathcal{C}_{\mathfrak{a} \mathfrak{b}}=\left\{|c|:\left(\begin{array}{l}
* * \\
c *
\end{array}\right) \in \sigma_{\mathfrak{a}}{ }^{-1} \Gamma \sigma_{\mathfrak{b}}, c \neq 0\right\} .
$$

As described in [11, Sections 2.5, 2.6] and [22, Lemma 1.25], there is a smallest element in the set $\mathcal{C}_{\mathfrak{a} \mathfrak{b}}$ which we shall denote by $c(\mathfrak{a}, \mathfrak{b})$. With this, we let

$$
c_{\Gamma}:=\max _{\mathfrak{a}, \mathfrak{b}}\left\{c(\mathfrak{a}, \mathfrak{b})^{-2}\right\} .
$$

An important point, shown in [11, Section 2.4], is that for any $\gamma \in \Gamma$

$$
\sigma_{\mathfrak{a}}{ }^{-1} \gamma \sigma_{\mathfrak{b}}=\left(\begin{array}{l}
* * \\
0
\end{array}\right) \Longrightarrow \mathfrak{a}=\mathfrak{b} \text { and } \sigma_{\mathfrak{a}}{ }^{-1} \gamma \sigma_{\mathfrak{b}}=\left(\begin{array}{ll}
1 & l \\
0 & l
\end{array}\right)
$$

for some $l \in \mathbf{R}$. The next result is similar to the second part of [22, Lemma 1.25].

Lemma A.1. For all $z \in \mathbf{H}$ and every cusp $\mathfrak{b}$

$$
y_{\Gamma}\left(\sigma_{\mathfrak{b}} z\right) \leqslant\left(c_{\Gamma}+1 / c_{\Gamma}\right)(y+1 / y) .
$$

Proof. We first prove the following fact about the invariant height function $y_{\Gamma}(w)$ : If $y_{\Gamma}(w)>Y$ then, for any cusp $\mathfrak{b}$, either $\operatorname{Im}\left(\sigma_{\mathfrak{b}}{ }^{-1} w\right)>Y$ or $\operatorname{Im}\left(\sigma_{\mathfrak{b}}{ }^{-1} w\right)<c_{\Gamma} / Y$. To see why this is true note that $y_{\Gamma}(w)>Y$ implies that there exists $\mathfrak{a}$ and $\gamma \in \Gamma$ so that $\operatorname{Im}\left(\sigma_{\mathfrak{a}}{ }^{-1} \gamma w\right)>Y$. Hence

$$
\left|j\left(\sigma_{\mathfrak{a}}{ }^{-1} \gamma \sigma_{\mathfrak{b}}, z\right)\right|^{2}<\frac{y}{Y}
$$

for $z=\sigma_{\mathfrak{b}}{ }^{-1} w$. Let $\gamma^{\prime}={\sigma_{\mathfrak{a}}}^{-1} \gamma \sigma_{\mathfrak{b}}=\left(\begin{array}{c}* * \\ c\end{array}\right)$. Now if $c=0$ then (A.2) implies that $\gamma^{\prime}=\left(\begin{array}{ll}1 & * \\ 0 & 1\end{array}\right)$ and therefore $\operatorname{Im}\left(\sigma_{\mathfrak{b}}{ }^{-1} w\right)>Y$. Otherwise, if $c \neq 0$, we have from (A.3) that

$$
|c y|^{2}<\frac{y}{Y}
$$


and $y<1 /\left(|c|^{2} Y\right)$. That implies $y<c_{\Gamma} / Y$ as required.

The proof of the lemma is now straightforward. The contrapositive of the first result is that if there exists a cusp $\mathfrak{b}$ so that

$$
c_{\Gamma} / Y \leqslant \operatorname{Im}\left(\sigma_{\mathfrak{b}}{ }^{-1} w\right) \leqslant Y
$$

then $y_{\Gamma}(w) \leqslant Y$. In other words, for any cusp $\mathfrak{b}$,

$$
c_{\Gamma} / Y \leqslant y \leqslant Y \Longrightarrow y_{\Gamma}\left(\sigma_{\mathfrak{b}} z\right) \leqslant Y .
$$

Choosing $Y=\left(c_{\Gamma}+1 / c_{\Gamma}\right)(y+1 / y)$ in (A.4) finishes the proof.

For completeness we also prove a lower bound for the invariant height of any point $z$ in $\mathbf{H}$.

Lemma A.2. For every $z \in \mathbf{H}$

$$
y_{\Gamma}(z) \geqslant\left\{\begin{array}{lll}
c_{\Gamma} & \text { if } & c_{\Gamma} \leqslant 1 / 2 \\
\sqrt{c_{\Gamma}-1 / 4} & \text { if } & c_{\Gamma}>1 / 2
\end{array}\right.
$$

Proof. Suppose $y_{\Gamma}(w)<Y$ then, for all cusps $\mathfrak{a}, \mathfrak{b}$ and all $\gamma \in \Gamma$ we have $\operatorname{Im}\left({\sigma_{\mathfrak{a}}}^{-1} \gamma \sigma_{\mathfrak{b}} z\right)<Y$ for $z=\sigma_{\mathfrak{b}}{ }^{-1} w$. Hence

$$
\left|j\left(\sigma_{\mathfrak{a}}{ }^{-1} \gamma \sigma_{\mathfrak{b}}, z\right)\right|^{2}>\frac{y}{Y} .
$$

We need to make a careful choice of $\mathfrak{a}, \mathfrak{b}$ and $\gamma$ to get the desired bounds. First choose $\mathfrak{a}, \mathfrak{b}$ and $\gamma_{0}$ so that $\sigma_{\mathfrak{a}}{ }^{-1} \gamma_{0} \sigma_{\mathfrak{b}}=\left(\begin{array}{cc}* * \\ c & d\end{array}\right)$ with $c_{\Gamma}=1 /|c|^{2}$. Recall that $\Gamma_{\mathfrak{b}}$, the subgroup of $\Gamma$ consisting of elements that fix $\mathfrak{b}$, is generated by a primitive element $\gamma_{\mathfrak{b}}$ that satisfies $\sigma_{\mathfrak{b}}{ }^{-1} \gamma_{\mathfrak{b}}^{l} \sigma_{\mathfrak{b}}=\left(\begin{array}{ll}1 & l \\ 0 & 1\end{array}\right)$. Hence

$$
\sigma_{\mathfrak{a}}{ }^{-1} \gamma_{0} \gamma_{\mathfrak{b}}^{l} \sigma_{\mathfrak{b}}=\left(\begin{array}{cc}
* & * \\
c & d+c l
\end{array}\right) .
$$

Choose $l$ so that $|x+d / c+l| \leqslant 1 / 2$ and put $\gamma=\gamma_{0} \gamma_{\mathfrak{b}}^{l}$ in (A.5) to get

$$
\left|j\left(\sigma_{\mathfrak{a}}{ }^{-1} \gamma \sigma_{\mathfrak{b}}, z\right)\right|^{2}=|c|^{2}\left(|x+d / c+l|^{2}+y^{2}\right)>\frac{y}{Y}
$$

and therefore

$$
1 / 4>\frac{c_{\Gamma} y}{Y}-y^{2} .
$$

Complete the square in (A.6) and replace $Y$ by $c_{\Gamma} / \sqrt{u^{2}+1}$ for a new parameter $u$ to get

$$
y=\operatorname{Im}\left(\sigma_{\mathfrak{b}}{ }^{-1} w\right)>\left(u+\sqrt{u^{2}+1}\right) / 2 .
$$

Since $y_{\Gamma}(w) \geqslant \operatorname{Im}\left({\sigma_{\mathfrak{b}}}^{-1} w\right)$, our work to this point has shown that

$$
y_{\Gamma}(w)<\frac{c_{\Gamma}}{\sqrt{u^{2}+1}} \Longrightarrow y_{\Gamma}(w)>\left(u+\sqrt{u^{2}+1}\right) / 2
$$

for any $u \geqslant 0$.

If $c_{\Gamma} \leqslant 1 / 2$ then taking $u=0$ in (A.7) shows that $y_{\Gamma}(w)<c_{\Gamma}$ is impossible. If $c_{\Gamma}>1 / 2$ then

$$
\frac{c_{\Gamma}}{\sqrt{u^{2}+1}}=\left(u+\sqrt{u^{2}+1}\right) / 2
$$

has a positive solution, namely $u=\left(2 c_{\Gamma}-1\right) / \sqrt{4 c_{\Gamma}-1}$. Substituting this value into (A.7), we arrive at the bound

$$
y_{\Gamma}(w)<\sqrt{c_{\Gamma}-1 / 4} \Longrightarrow y_{\Gamma}(w)>\sqrt{c_{\Gamma}-1 / 4}
$$

and deduce that $y_{\Gamma}(w)<\sqrt{c_{\Gamma}-1 / 4}$ is impossible. This completes the proof.

Let $y_{\Gamma}$ be the minimum value of $y_{\Gamma}(z)$. For example, when $\Gamma=\mathrm{SL}_{2}(\mathbf{Z})$ we have $c_{\Gamma}=1$ and therefore by Lemma A. 2 we know $y_{\Gamma} \geqslant \sqrt{3} / 2$. In fact, by examining the Ford fundamental domain for $\mathrm{SL}_{2}(\mathbf{Z})$ we see that $y_{\Gamma}=\sqrt{3} / 2$.

We conclude with the result quoted in the proof of Proposition 3.3. 
Lemma A.3. For $\operatorname{Re}(s)=\sigma>1$ and an implied constant depending on $\sigma, \Gamma$ alone,

$$
E_{\mathfrak{a}}(z, s)-\operatorname{Im}\left(\sigma_{\mathfrak{a}}{ }^{-1} z\right)^{s} \ll y_{\mathbf{F}}(z)^{1-\sigma} .
$$

Proof. We know from [11, Corollary 3.5] that

$$
E_{\mathfrak{a}}\left(\sigma_{\mathfrak{b}} z, s\right)=\delta_{\mathfrak{a} \mathfrak{b}} y^{s}+\phi_{\mathfrak{a} \mathfrak{b}}(s) y^{1-s}+O\left(e^{-2 \pi y}\right)
$$

as $y \rightarrow \infty$. Hence

$$
E_{\mathfrak{a}}\left(\sigma_{\mathfrak{a}} z, s\right)-\operatorname{Im}\left(\sigma_{\mathfrak{a}}{ }^{-1} \sigma_{\mathfrak{a}} z\right)^{s} \ll y^{1-\sigma}
$$

as $y \rightarrow \infty$ and, for $\mathfrak{a} \neq \mathfrak{b}$

$$
\begin{aligned}
E_{\mathfrak{a}}\left(\sigma_{\mathfrak{b}} z, s\right)-\operatorname{Im}\left(\sigma_{\mathfrak{a}}{ }^{-1} \sigma_{\mathfrak{b}} z\right)^{s} & =-\operatorname{Im}\left(\sigma_{\mathfrak{a}}{ }^{-1} \sigma_{\mathfrak{b}} z\right)^{s}+\phi_{\mathfrak{a} \mathfrak{b}}(s) y^{1-s}+O\left(e^{-2 \pi y}\right) \\
& \ll \operatorname{Im}\left(\sigma_{\mathfrak{a}}{ }^{-1} \sigma_{\mathfrak{b}} z\right)^{\sigma}+y^{1-\sigma} .
\end{aligned}
$$

By (A.2) we have $\sigma_{\mathfrak{a}}{ }^{-1} \sigma_{\mathfrak{b}}=\left(\begin{array}{cc}* & * \\ c & d\end{array}\right)$ with $c \neq 0$ and

$$
\operatorname{Im}\left(\sigma_{\mathfrak{a}}{ }^{-1} \sigma_{\mathfrak{b}} z\right)^{\sigma}=\frac{y^{\sigma}}{|c z+d|^{2 \sigma}}=\frac{y^{\sigma}}{\left(|c x+d|^{2}+|c y|^{2}\right)^{\sigma}} \leqslant \frac{y^{\sigma}}{|c y|^{2 \sigma}} \leqslant\left(c_{\Gamma}\right)^{\sigma} y^{-\sigma} .
$$

The result follows.

\section{References}

[1] G. Chinta, N. Diamantis, and C. O'Sullivan. Second order modular forms. Acta Arith., 103:209-223, 2002.

[2] G. Chinta and C. O'Sullivan. Optimal bounds for period polynomials. Preprint, 2005.

[3] Y. Choie and N. Diamantis. Rankin-Cohen brackets on higher order modular forms. Preprint, 2006.

[4] N. Diamantis, M. Knopp, G. Mason, and C. O'Sullivan. L-functions of second-order modular forms. Preprint, 2005.

[5] N. Diamantis and C. O'Sullivan. Hecke theory of series formed with modular symbols and relations among convolution L-functions. Math. Annalen, 318:85-105, 2000.

[6] D. Goldfeld. Modular elliptic curves and Diophantine problems. Number Theory (Banff, AB, 1988), pages 157-175, 1990.

[7] D. Goldfeld. The distribution of modular symbols. In Number theory in progress (Zakopane-Kościelisko, 1997), volume 2, pages 849-865, Berlin, 1999. de Gruyter.

[8] D. Goldfeld. Zeta functions formed with modular symbols. In Automorphic forms, automorphic representations, and arithmetic (Fort Worth, TX, 1996), volume 66 of Proc. Sympos. Pure Math., Providence, RI, 1999. Amer. Math. Soc.

[9] D. Goldfeld. Modular forms, elliptic curves, and the abc-conjecture. In A panorama in number theory: The view from Baker's garden. Based on a conference in honor of Alan Baker's 60th birthday, pages 128-147. Cambridge University Press, Cambridge, 2002.

[10] D. Goldfeld and C. O'Sullivan. Estimating additive character sums for Fuchsian groups. Ramanujan J., 7:241-267, 2003.

[11] H. Iwaniec. Spectral methods of automorphic forms, volume 53 of Graduate studies in mathematics. Amer. Math. Soc., second edition, 2002.

[12] J. Jorgenson and C. O'Sullivan. Convolution Dirichlet series and a Kronecker limit formula for secondorder Eisenstein series. Nagoya Math. J., 179:47-102, 2005.

[13] J. Jorgenson and C. O'Sullivan. Dedekind sums from third-order Eisenstein series. Preprint, 2006. 
[14] P. Kleban and D. Zagier. Crossing probabilities and modular forms. J. Stat. Phys., 113:431-454, 2003.

[15] M. Knopp and G. Mason. Generalized modular forms. J. Number Theory, 99:1-28, 2003.

[16] M. Knopp and G. Mason. Vector-valued modular forms and Poincaré series. Illinois J. of Math., 48(4):1345-1366, 2004.

[17] C. O'Sullivan. Properties of Eisenstein series formed with modular symbols. J. Reine Angew. Math, 518:163-186, 2000.

[18] Y. N. Petridis. Spectral deformations and Eisenstein series associated with modular symbols. Int. Math. Res. Not., 19:991-1006, 2002.

[19] Y. N. Petridis and M. S. Risager. Modular symbols have a normal distribution. Geom. Funct. Anal., 14:1013 - 1043, 2004.

[20] M. S. Risager. Automorphic forms and modular symbols. PhD thesis, University of Aarhus, 2003.

[21] A. Selberg. On the estimation of Fourier coefficients of modular forms. In Proc. Symposia in Pure Math., volume 8, pages 1-15. AMS, Providence, 1965.

[22] G. Shimura. Introduction to the Arithmetic Theory of Automorphic Functions. Princeton Univ. Press, Princeton, NJ, 1971.

[23] K. Taylor. Analytic continuation of vector-valued nonanalytic Eisenstein series. PhD thesis, Temple University, 2006.

\author{
Jay Jorgenson \\ Department of Mathematics \\ City College of New York \\ Convent Avenue at 138th Street \\ New York, NY 10031 \\ e-mail: jjorgenson@mindspring.com \\ Cormac O'Sullivan \\ Department of Mathematics \\ Bronx Community College \\ University Avenue and West 181st Street \\ Bronx, NY 10453 \\ e-mail: cormac12@juno.com
}

